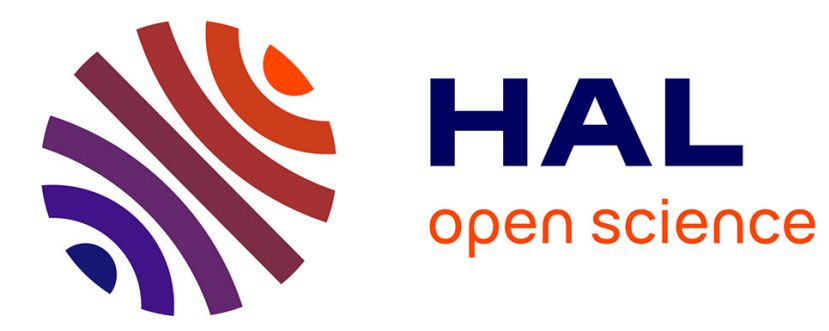

\title{
Expériences de laboratoire en économie et incitations monétaires
}

\author{
Nathalie Etchart-Vincent
}

\section{To cite this version:}

Nathalie Etchart-Vincent. Expériences de laboratoire en économie et incitations monétaires. Revue d'Economie Politique, 2006, 116 (3), pp.383-418. hal-00395894

\section{HAL Id: hal-00395894 \\ https://hal.science/hal-00395894}

Submitted on 18 Oct 2012

HAL is a multi-disciplinary open access archive for the deposit and dissemination of scientific research documents, whether they are published or not. The documents may come from teaching and research institutions in France or abroad, or from public or private research centers.
L'archive ouverte pluridisciplinaire HAL, est destinée au dépôt et à la diffusion de documents scientifiques de niveau recherche, publiés ou non, émanant des établissements d'enseignement et de recherche français ou étrangers, des laboratoires publics ou privés. 
Expériences de laboratoire en économie et incitations monétaires

Experimenting in the Lab in Economics and Monetary Incentives

\author{
Nathalie Etchart-Vincent
}

CNRS, CIRED*

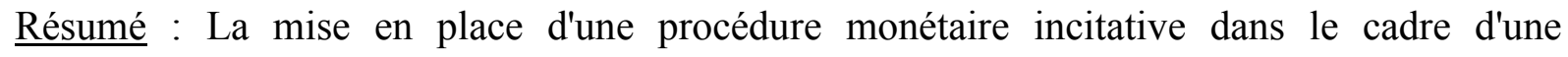
expérience de laboratoire en économie suppose l'existence d'une relation positive étroite entre incitation et effort d'une part et entre effort et performance d'autre part. Pourtant, sur le plan empirique, l'impact des incitations monétaires sur l'effort et/ou la performance apparaît plus mitigé, voire négatif. Nous revenons ici sur un certain nombre d'arguments théoriques et empiriques avancés dans la littérature pour expliquer cet écart. Sont ainsi mis en cause le montant insuffisant des incitations, l'inadéquation des procédures incitatives utilisées, la fragilité du lien entre incitation et effort et/ou entre effort et performance, le rôle ambigu de la motivation intrinsèque. Nous nous intéressons ensuite au cas particulier de l'expérimentation dans des contextes de pertes, qui pose de manière plus fondamentale la question de l'opportunité des incitations monétaires. Nous concluons sur la nécessité d'une utilisation pragmatique de ces dernières selon la nature de l'étude envisagée.

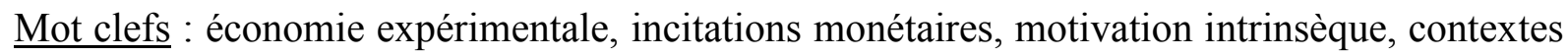
de pertes

Abstract: A strong positive relation is generally assumed between monetary incentives and effort on the one hand, and between effort and performance on the other hand, which

\footnotetext{
* Chargée de Recherche au CNRS. Adresse : Centre International de Recherche sur l'Environnement et le Développement (CNRS/EHESS), Jardin Tropical, 45 bis, Av. de la Belle Gabrielle, 94736 Nogent sur Marne ; email : etchart@centre-cired.fr. Je remercie les deux rapporteurs anonymes pour leurs très judicieuses remarques
} 
legitimates the introduction of monetary incentives in economic laboratory experiments. Still, the empirical impact of incentives on effort and/or performance appears to be rather mixed or even negative. We examine the ins and outs of the debate since they all are, in a more or less radical manner, an invitation to a more pragmatic attitude towards incentives. We first show that embarrassing empirical results cannot be ascribed to an alleged inappropriateness of the incentive procedure (as regards the level of incentives or the procedure itself). We then examine the robustness of the relation between incentives, effort, and performance and elaborate upon the reasons why incentives sometimes do not enhance (or even damage) effort and/or why effort sometimes does not induce better performance (whether it be ineffective or harmful upon it). Next, we discuss the peculiar case of experimentation in the loss domain, which raises the more fundamental question of whether monetary incentives can be introduced. We finally conclude on the need of a pragmatic use of monetary incentives, depending on the very features of the experimental design (including subjects' cognitive and psychological characteristics).

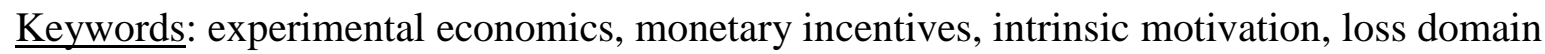

JEL : B41, C90, C91

et suggestions, qui m'ont permis d'améliorer considérablement le texte. Je suis seule responsable des éventuelles erreurs qui pourraient subsister. 


\section{Introduction}

Le recours à la méthode expérimentale en économie s'est largement développé ces dernières années, notamment par le biais d'études de laboratoire ${ }^{1}$. L'expérimentation en laboratoire est une méthodologie fondée sur la reconstitution in vitro d'une situation économique simplifiée dont les variables sont contrôlées par l'expérimentateur, ce qui d'une part permet l'interprétation la plus univoque possible des résultats et d'autre part assure la reproductibilité du protocole. Elle constitue un outil d'investigation empirique précieux, utilisé aussi bien à des fins d'évaluation du pouvoir descriptif des modèles (dont elle permet de tester empiriquement les prédictions), que, plus généralement, comme outil d'investigation des comportements économiques et, enfin, comme support de recommandations prescriptives à l'usage des décideurs publics (Roth [1988]). Comme nous l'avons montré ailleurs, la montée en puissance de la méthode expérimentale en économie s'accompagne de critiques plus ou moins radicales (Etchart-Vincent [2005]). En tout état de cause, certaines précautions doivent être prises pour permettre la collecte de données de bonne qualité, mais la nature de ces précautions fait toujours débat. L'un des points les plus discutés au cours des 30 dernières années concerne ce que l'on a appelé les 'incitations monétaires' (monetary incentives en anglais). C'est à ces dernières que nous nous intéressons $\mathrm{ici}^{2}$.

\footnotetext{
${ }^{1}$ Notamment, car il existe également une expérimentation de terrain et une expérimentation naturelle. Dans le premier cas, il s'agit de dépasser la critique faite aux expériences de laboratoire concernant leur éventuel irréalisme, en testant le comportement d'individus 'réels' dans des situations de choix 'réelles', face à des biens 'réels'. Dans le second cas, c'est la réalité elle-même qui constitue un laboratoire vivant parce que, dans des contextes évidemment très particuliers, elle offre spontanément le contrôle requis sans qu'une expérience soit explicitement mise en place (c'est le cas par exemple lorsqu'une mesure de politique publique est introduite, toutes choses égales par ailleurs). Nous renvoyons à Harrison et List [2004] pour une comparaison détaillée des différentes modalités d'expérimentation.

${ }^{2}$ Il existe bien évidemment des formes non monétaires d'incitation (voir par exemple Bénabou et Tirole [2003]). On opposera les incitations du type 'carotte' (délégation, autonomie ...) et celles du type 'bâton' (surveillance, sanction...) et, au sein de chaque catégorie, les incitations explicites et celles implicites (regards plus ou moins bienveillants, ostracisme ...). Dans le cadre de l'expérimentation, ce sont les incitations monétaires - 'carottes' explicites - qui prévalent. Il faut cependant se méfier des incitations implicites, qui ne sont pas introduites volontairement par l'expérimentateur mais peuvent se manifester au cours de l'expérience et affecter les résultats (par exemple lorsqu'un sujet ne répond pas assez vite et subit le regard agacé des autres sujets).
} 
Les incitations monétaires sont bien des incitations (au sens que la théorie des incitations donne à ce terme). Il s'agit en effet d'une technique de rémunération qui fait dépendre cette dernière de la performance du sujet et incite donc celui-ci à prendre les décisions qui correspondent à ses véritables préférences de façon à maximiser son gain (ou minimiser sa perte, selon le cas). La procédure incitative faisant de la révélation des véritables préférences une stratégie optimale pour le sujet, on peut, dans la terminologie des la théorie des contrats, la qualifier de mécanisme révélateur. Le postulat sous-jacent est qu'en l'absence d'une telle procédure, le sujet choisirait plutôt de minimiser son effort et donc éventuellement de répondre n'importe quoi - ce qui aurait pour conséquence de nuire à la qualité et à la fiabilité des données ainsi collectées.

Le principe même de la mise en place d'une procédure incitative repose sur le postulat d'une relation étroite entre incitation et effort d'une part (sans incitations, pas d'effort) puis entre effort et performance (l'effort du sujet garantit la qualité de ses réponses et donc celle des données recueillies). La relation incitation - effort - performance est pourtant loin d'être triviale. En effet, autant il est aisé de concevoir la nature de cette relation et de donner un contenu précis aux notions d'effort et de performance dans le cadre de relations contractuelles établies (sur le marché du travail par exemple), autant il peut être délicat de mesurer empiriquement l'effort fourni et, surtout, d'identifier une norme de performance dans le cadre d'une expérimentation.

Bonner et Sprinkle [2002] décomposent l'effort en ses composantes (direction, durée, intensité notamment), mais il faut aussi le définir par sa nature, qui en l'occurrence est ici cognitive et motivationnelle (effort intellectuel de concentration, de compréhension, de réflexion, etc.). Si la notion d'effort est théoriquement identifiable, la question se pose de sa mesure dans le cadre d'une expérience : comment évaluer le degré de concentration du sujet ou l'effort de compréhension qu'il fournit ? Les mesures de cohérence qui sont effectuées dans la plupart 
des travaux expérimentaux ${ }^{3}$ visent à évaluer la fréquence des erreurs liées à un effort insuffisant de la part du sujet. Mais en pratique, il est difficile de distinguer ce type d'erreurs de celles liées aux limitations cognitives intrinsèques du sujet.

La notion de performance est plus difficile encore à circonscrire. Les études compilées par Camerer et Hogarth [1999] et Hertwig et Ortmann [2001] permettent de dresser une liste à la Prévert des indicateurs de performance susceptibles d'être retenus : le respect de tel ou tel axiome, la capacité de mémorisation, la quantité de travail effectuée, la rapidité des réponses, la capacité à résoudre le problème posé, le niveau d'erreur ${ }^{4}$, le caractère bayésien des comportements, le degré de convergence vers l'équilibre, le degré d'ancrage sur des croyances initiales, la sujétion aux effets de présentation, le degré de confiance dans les décisions prises, etc. Notons qu'on trouve ici aussi bien des principes de rationalité économique au sens strict que des critères de 'bon sens'.

Dans ce cadre d'analyse, il est possible d'étudier l'impact en termes de performance d'une modification des conditions de rémunération. Par exemple, si les sujets non incités monétairement répondent moins rapidement ou violent davantage la dominance stochastique que ceux qui le sont, on en déduit que la performance pâtit de l'absence d'incitation. Cependant, cette approche repose sur le postulat que des réponses qui violent des principes de rationalité traduisent une faible performance du sujet. Que dire lorsque de telles déviations sont mises en évidence de manière répétée et systématique à partir de protocoles expérimentaux différents et, surtout, qu'elles sont également présentes dans le monde économique réel (sur les marchés financiers par exemple ; cf Shapira [2000]) ? Il est alors difficile de considérer que la "norme" est donnée par la conformité aux axiomes ou aux principes de rationalité standards - et de mettre en cause l'absence ou l'insuffisance des

\footnotetext{
${ }^{3}$ Des questions identiques sont posées à deux reprises au cours de l'expérience ; la comparaison des réponses permet de mesurer le degré de cohérence des sujets.

${ }^{4}$ Dont on voit qu'il est un indicateur aussi bien d'effort que de performance, ce qui est intellectuellement peu satisfaisant dès lors que l'on souhaite interroger le lien entre effort et performance.
} 
incitations monétaires utilisées dans le cadre de l'expérimentation. Mieux, ce sont les incitations qui s'avèrent parfois artificielles, lorsqu'elles perturbent les intentions et les préférences des sujets et se traduisent par l'adoption de comportements plus rationnels que ceux qui prévaudraient dans la vie. En outre, lorsque les indicateurs de rationalité se contredisent (par exemple, lorsqu'une rapidité de réponse accrue va de pair avec une moindre cohérence), lequel retenir pour évaluer l'incidence des incitations monétaires sur la performance ? Enfin, la définition d'une norme de performance est plus délicate encore lorsque l'expérience ne vise qu'à éliciter les préférences des individus : comment mesurer la performance lorsque la variable étudiée est le degré d'aversion au risque de l'individu ou le nombre d'achats qu'il effectue ?

Cette ambiguïté autour des indicateurs de performance et de leur mesure rend parfois délicate l'interprétation des résultats empiriques, en même temps qu'elle contribue à alimenter les débats méthodologiques autour de la question des incitations monétaires. En effet, un même résultat peut signifier une chose et son contraire. Si l'on considère que l'individu est performant lorsqu'il viole les axiomes de rationalité standard parce que c'est ainsi qu'il se comporte dans la vie réelle, alors les incitations vont être considérées comme nuisibles à la performance si elles augmentent de manière artificielle le degré de rationalité. En revanche, un impact similaire sur le degré de rationalité sera considéré comme positif si la norme de performance est donnée par le taux de conformité aux axiomes standards ${ }^{6}$. L'interprétation des données peut ainsi être paradoxale ${ }^{7}$.

\footnotetext{
${ }^{5}$ Il est à cet égard symptomatique que les méta-analyses excluent les études qui ne comportent pas de standard de performance explicite (voir par exemple Bonner et Sprinkle [2002]).

${ }^{6}$ Les enjeux stratégiques autour de l'interprétation des résultats sont importants. L'idée selon laquelle l'existence de "paradoxes" expérimentaux est liée à l'absence d'incitations (qui empêche les individus de se comporter selon leurs véritables préférences, c'est-à-dire, en l'occurrence, de se conformer aux principes de rationalité) s'inscrit dans une stratégie immunisatrice, qui cherche à discréditer le recours à l'expérimentation pour minimiser la portée des résultats "gênants" (voir Etchart-Vincent [2005]).

${ }^{7}$ Nous illustrerons notre propos par deux exemples précis. Le premier est emprunté à Harless et Camerer [1994]. Ces derniers montrent que les données obtenues à partir de choix réels présentent des taux d'erreur plus faibles. Du coup, cette réduction dans la variance des réponses tend à accroître la signification statistique des 'anomalies' constatées (par rapport au modèle de rationalité standard), ce qui amène paradoxalement à conclure à une baisse
} 
Notons qu'un débat s'est développé, notamment au sein de la communauté des psychologues, concernant la pertinence des indicateurs d'effort ou de performance retenus. Les conclusions des uns sont ainsi parfois remises en cause, à la faveur d'une interprétation différente, par les autres (voir par exemple le débat, par articles interposés, entre Deci, Koestner et Ryan [1999a] [1999b] d'un côté et Eisenberger et Armeli [1997] ; Eisenberger et Cameron [1996] [1998] ; Eisenberger, Pierce et Cameron [1999], Eisenberger, Rhoades et Cameron [1999] ; Lepper, Henderlong et Gingras [1999] de l'autre ; voir aussi, par exemple, la méta-analyse de Kunz et Pfaff $\left.[2002]^{8}\right)$.

Même si l'on ne retient que les études empiriques les moins ambiguës, c'est-à-dire celles dans lesquelles il est possible d'identifier de manière univoque une norme de performance et de mesurer l'impact des incitations monétaires sur cette dernière (voir les méta-analyses de Camerer et Hogarth [1999], Hertwig et Ortmann [2001], Bonner et Sprinkle [2002]), il apparaît que cet impact est loin d'être systématiquement positif. En fait, il est souvent nul et parfois même négatif. Deux types d'arguments ont alors été avancés pour expliquer ces résultats. Le premier argument, conservateur, met en cause la manière dont les incitations sont mises en place - leur montant est trop faible ou la procédure incitative inadaptée - pour expliquer leur inefficacité (c'est-à-dire leur incapacité à générer un effort soutenu et donc une performance élevée) et réaffirmer leur nécessité. Le second argument, à la fois plus profond et plus nuancé dans ses conclusions, fait dépendre de la nature de l'expérience (tâche à accomplir et environnement expérimental) et des caractéristiques psychologiques et

de la performance si cette dernière est définie comme la conformité à cette rationalité standard. Un second exemple paradoxal est fourni par Grether et Plott [1979]. Il apparait qu'en stimulant l'effort (la concentration) du sujet, les incitations monétaires vont amener ce dernier à effectuer des choix qui seront certes plus proches de ses véritables préférences mais aussi moins conformes à la rationalité standard. Selon la définition retenue pour la performance, on conclura alors à une efficacité ou à une perversité des incitations.

${ }^{8}$ A l'issue d'une relecture minutieuse des études de Eisenberger et Cameron [1996] et Deci, Koestner et Ryan [1999a], Kunz et Pfaff [2002] estiment que les résultats de ces derniers sont finalement moins défavorables (que ne le souhaiteraient les auteurs) aux incitations monétaires. 
cognitives de l'être humain l'efficacité des incitations monétaires et donc le caractère opportun ou non de la mise en place d'un système incitatif.

L'objet de cet article est de faire le point sur les enjeux du débat théorique et empirique autour des incitations monétaires et de mettre à plat les différents arguments avancés. Le papier est organisé comme suit. Nous partons de la position de principe, adossée au concept d'homo oeconomicus, selon laquelle les incitations monétaires sont nécessaires pour produire des données de bonne qualité, avant de recenser un certain nombre de résultats empiriques concernant l'impact des incitations sur la performance (section 2). Si certains de ces résultats sont conformes aux prédictions, un grand nombre d'entre eux s'avèrent plus mitigés (les incitations apparaissant comme inutiles) voire négatifs (les incitations monétaires s'avérant nuisibles). Il s'agit alors d'expliquer pourquoi la relation incitation - effort - performance n'est pas aussi étroite et/ou positive que prévu. Nous nous intéressons tout d'abord aux arguments qui ont été avancés pour expliquer pourquoi les incitations s'avèrent parfois inefficaces. Un premier argument invoque le caractère prétendument inadéquat des procédures utilisées en pratique pour réaffirmer le caractère nécessaire des incitations monétaires (section 3), tandis que le second souligne la distension du lien entre incitation et effort et/ou entre effort et performance et conclut au caractère inutile des incitations dans certains cas (section 4). Nous étudions ensuite les raisons pour lesquelles les incitations peuvent être nuisibles, lorsqu'elles réduisent l'effort (en détruisant la motivation intrinsèque notamment) ou lorsque l'effort accru nuit à la performance (section 5). Nous nous attardons enfin sur un cas particulier, celui de l'expérimentation dans les pertes, qui pose de manière plus fondamentale la question de la possibilité même de recourir aux incitations monétaires (section 6). Ce sera alors pour nous l'occasion de conclure, de manière pragmatique, sur l'opportunité de la mise en place d'un système incitatif (section 7). 


\section{La relation théorique rétribution monétaire-effort-performance et sa pertinence empirique}

Nous commençons par présenter l'approche traditionnelle en économie (même si elle est de moins en moins monopolistique) en ce qui concerne la rémunération des sujets au cours d'une expérience (2.1.), avant d'évaluer la validité empirique de cette approche (2.2.).

\section{1. La relation théorique rémunération - effort - performance et l'homo} oeconomicus

Nous distinguons les deux types de rémunérations possibles - fixe et variable - de façon à expliciter la fonction de chacune d'entre elles et le rôle particulier joué par la rémunération variable, autrement appelée incitation monétaire.

\subsubsection{La rémunération fixe}

On peut envisager un premier type de rémunération, inconditionnel et proposé au sujet pour le remercier de sa participation. Cette rémunération est donc forfaitaire et ne dépend pas du comportement du sujet. On distingue deux fonctions à cette forme de rémunération. D'une part, le souci de dédommager l'individu de son effort (désutilité du trajet ou du temps passé et de l'énergie dépensée à faire l'expérience par exemple) et d'autre part celui de s'attirer ses bonnes grâces en créant chez lui une sorte de dette morale. La première motivation s'inscrit dans une approche beckerienne : la rétribution doit être proportionnelle à l'effort fourni, à la longueur de la tâche et à sa pénibilité. La seconde se conçoit plutôt dans une logique de réciprocité ou de don-contre don (Akerlof [1984]). L'idée sous-jacente est que, face à des tâches longues et/ou cognitivement ardues et fatigantes, la rétribution va permettre de soutenir la mobilisation des sujets tout au long de l'expérience : parce qu'ils sont rémunérés, les sujets 
ne vont pas oser tirer au flanc, même si la rétribution est indépendante de leur comportement. Nous donnons simplement deux exemples attestant de la plausibilité psychologique de cette hypothèse. Tout d'abord, étudiant quelle modalité - entre le fait de leur verser une rémunération tous les mois et celui de les garder en contact avec les services sociaux (de façon à les maintenir informés et à leur rappeler de mettre à jour leur vaccination) - serait la plus efficace pour inciter des drogués qui ont déjà reçu une première injection du vaccin contre l'hépatite B à recevoir les deux dernières injections, Seal et al. [2003] montrent que la rétribution monétaire s'avère trois fois plus efficace que la simple information. Dans le même ordre d'idée, Jobber, Saunders et Mitchell [2004] établissent que l'impact d'une rémunération préalable sur le taux de réponse à un questionnaire est significatif. Plus le prépaiement est élevé, plus le taux de réponse est élevé, comme si les individus étaient gênés de recevoir de l'argent sans remplir leur part du contrat (moral) : est ainsi provoquée chez eux une obligation de réciprocité.

Notons cependant que, dans le cadre d'une enquête comme d'une expérience, le fait de répondre est une chose, celui de bien répondre en est une autre. On peut ainsi considérer que la simple rémunération forfaitaire est insuffisante en ne suscitant qu'une adhésion et un effort de façade. C'est bien la raison pour laquelle on distingue la rémunération "tout court" de la rémunération à la performance, censée influer sur la qualité des réponses et considérée comme indispensable par la majorité des économistes.

\subsubsection{La rémunération variable : l'incitation monétaire}

Ainsi que le soulignent Kagel et Roth [1995], le choix de recourir à des décisions réelles (avec incitations monétaires) ou hypothétiques (avec rémunération forfaitaire uniquement) trace la ligne de partage entre les études publiées dans les revues d'économie et celles publiées dans 
les revues de psychologie. Alors que les psychologues considèrent en général que la rémunération conditionnelle aux choix effectués est superflue (voire nuisible, comme on le verra plus loin), la majorité des économistes la tiennent pour nécessaire, en raison précisément de ses vertus incitatives. Elle est en effet susceptible d'affecter positivement à la fois le niveau d'effort fourni par le sujet et son état d'esprit lorsqu'il aborde l'expérience.

Les économistes ${ }^{9}$ font 1 'hypothèse que les sujets ne travaillent pas pour rien et que leur effort cognitif est une ressource rare qu'ils cherchent à allouer stratégiquement (Hertwig et Ortmann [2001]). Dans la vie réelle, l'effort est motivé par la perspective des gains censés en résulter et les incitations (sur le marché du travail par exemple) sont précisément là pour promouvoir effort et performance (Gibbons [1997] ; Lazear [2000]). Cette logique incitative doit donc se retrouver dans le laboratoire ${ }^{10}$, l'idée sous-jacente étant que si les sujets ne sont pas rémunérés de manière contingente à leur performance (ou si cette rémunération est insuffisante), ils risquent fort de ne pas mettre en œuvre un effort cognitif suffisant pour révéler leurs véritables préférences (Smith [1976] ; Smith et Levin [1996] ; Smith et Walker [1993]) auquel cas les données collectées n'auront aucune valeur. C'est Smith qui ouvre le débat en 1962 en présentant les résultats d'une étude expérimentale menée sur des marchés d'enchères et dans laquelle il apparaît que la convergence vers l'équilibre n'est possible qu'en présence d'incitations monétaires. De nombreux résultats expérimentaux contraires au modèle EU ont ainsi été déboutés a priori, leurs contempteurs arguant de l'insuffisance de la procédure incitative utilisée (Harrison [1994] ; Hirshleifer et Riley [1992] ; Smith et Walker [1993]). Nous renvoyons le lecteur à Bonner et Sprinkle [2002] pour une analyse détaillée des mécanismes motivationnels et cognitifs par lesquels les incitations monétaires sont censées

\footnotetext{
${ }^{9}$ Comme le souligne Camerer [1997], l'attitude officielle - monolithique - vis-à-vis des incitations dissimule en fait un certain scepticisme, qui se manifeste lors de discussions informelles. Cependant, depuis ses débuts (Wallis et Friedman [1942]), l'économie expérimentale fait de la mise en place d'incitations un passage obligé, presque un rite initiatique (voir Roth [1993] pour des détails historiques). Nous verrons plus loin que la force du rite s'est cependant assouplie ces dernières années.
} 
augmenter la performance en soutenant l'effort de l'individu (en l'amenant à se fixer des buts plus élevés ou à développer un intérêt accru pour la tâche, par exemple).

Par ailleurs, lorsqu'il s'agit de faire prendre au sujet des décisions de nature économique (décision d'investissement ou d'achat par exemple), le fait de le rémunérer en fonction de sa performance est censé l'aider sur le plan cognitif à dépasser le côté potentiellement artificiel et ludique de l'expérimentation en laboratoire et à raisonner comme il le ferait s'il était confronté à une même situation dans la vie réelle (à savoir en homo oeconomicus). Dans la mesure où les économistes sont avant tout intéressés par des décisions qui ne sont pas hypothétiques ${ }^{11}$, il est naturel de recourir à des incitations monétaires (Davis et Holt [1993]). On n'a plus besoin de supposer que les agents sont suffisamment motivés pour agir comme dans une situation réelle - ils sont dans une situation réelle, c'est-à-dire dotée d'enjeux réels (Ohana [2004]). On sort ainsi de la logique du "comme si". En particulier, parce qu'elle met le sujet en situation d'assumer financièrement les conséquences des décisions qu'il a prises, l'incitation monétaire permet de lutter contre la tentation que ce dernier pourrait avoir, par exemple, de ne chercher qu'à 'plaire' à l'expérimentateur (Gintis [2001]) ou de prendre des risques élevés 'juste pour voir'. Dans cette optique incitative, c'est la perspective d'une récompense qui va motiver l'individu, qui ne la percevra que s'il s'est "bien" comporté. Contrairement au type de rémunération évoqué précédemment, il s'agit donc d'une rétribution ex-post et conditionnelle.

$\mathrm{Au}$ total, ce sont donc deux types de rémunération qui se dessinent, dont l'objectif est visiblement distinct même s'ils sont tous deux susceptibles d'affecter l'effort fourni par le sujet et donc sa performance. Il s'agit d'une part de la rémunération récompense, dont la perception et le montant sont conditionnels à l'effort fourni, et d'autre part de la rémunération

\footnotetext{
${ }^{10}$ On mentionnera notamment le recours à des techniques qui, telle la procédure Becker-De Groot-Marshak, font de la révélation des véritables préférences une stratégie optimale pour l'individu et sont donc des mécanismes révélateurs au sens de la théorie des contrats.

${ }^{11}$ Notons que ce point de vue se discute : selon Kühberger [2001], les décisions économiques sont fondamentalement hypothétiques, puisqu'elles obligent l'individu à se projeter dans le futur et à anticiper des événements, des revenus ou des sentiments qu'il ne connaît pas encore.
} 
dédommagement, forfaitaire et inconditionnelle. Peu de travaux se sont intéressés à l'impact de la rémunération forfaitaire sur la performance dans le contexte expérimental ${ }^{12}$. Les incitations monétaires ont en revanche fait l'objet d'une littérature abondante, d'où il ressort qu'elles sont parfois efficaces, souvent inutiles et dans certains cas nuisibles.

\section{2. La réalité : un impact mitigé des incitations}

Nous commençons par recenser quelques travaux conformes aux prédictions théoriques fondées sur la rationalité de l'homo oeconomicus et évoquées précédemment, avant de donner un aperçu des résultats selon lesquels les incitations seraient au mieux inutiles, au pire nuisibles. En tout état de cause, comme en témoignent quelques méta-analyses récentes, une grande hétérogénéité ressort de la littérature. On se référera notamment à Camerer et Hogarth [1999], qui s'appuient sur 74 études expérimentales - publiées dans les plus grandes revues d'économie (American Economic Review, Econometrica, Quaterly Journal of Economics, Journal of Political Economy) et obéissant à un cahier des charges précis - pour dresser un bilan pour le moins mitigé des effets des incitations monétaires. On renvoie aussi à Hertwig et Ortmann [2001], qui recensent un certain nombre de travaux publiés dans le Journal of Behavioral Decision Making et dont les conclusions quant à l'impact des incitations sont également très disparates (voir Hertwig et Ortmann [2001] pp. 392-394 pour un tableau récapitulatif qui met en évidence cette diversité ${ }^{13}$ ). On se référera enfin avec intérêt à Bonner et al. [2000] et à Bonner et Sprinkle [2002] pour une recension similaire et des conclusions du même acabit, à partir d'une littérature diversifiée à dominante comptable.

\footnotetext{
${ }^{12}$ Citons l'étude de Deci, Koestner et Ryan [1999a], qui montre l'incidence négative de la rémunération sur la motivation intrinsèque et donc sur l'effort de l'individu.

${ }^{13}$ Les conclusions de Hertwig et Ortmann [2001] se situant dans la lignée de celles de Smith et Walker [1993] apparaissent plus favorables aux incitations. Mais outre que leur échantillon est plus réduit que celui de Camerer et Hogarth [1999] (10 études seulement, publiées dans une seule et même revue qui plus est, ce qui crée un biais d'échantillonnage), la teneur du message reste substantiellement la même.
} 


\subsubsection{Les incitations efficaces}

Il existe tout d'abord des résultats favorables à la mise en place d'incitations monétaires, ces dernières permettant à la fois la convergence des comportements vers l'optimalité économique et la réduction de leur variance (Hertwig et Ortmann [2001]). Par exemple, les incitations semblent réduire la fréquence du paradoxe d'Allais (Burke [1996]) et celle des renversements de préférences (Bohm [1994]). De même, Brookshire et Coursey [1987] et Coursey, Hovis et Schulze [1987] montrent que l'écart entre disposition à payer et disposition à recevoir est soluble dans les incitations (et l'apprentissage). Les incitations financières affecteraient donc positivement la performance (Davis et Holt [1993] ; Harrison [1992] ; Smith [1991]), par exemple en réduisant les effets de présentation (Levin, Chapman et Johnson [1988]), en améliorant la prise en compte du coût de l'information (van Wallendael et Guignard [1992]) ou en permettant la révélation de la véritable disposition à payer pour un bien environnemental (Harrison et Rutström [2004]). En outre, les incitations contribueraient à réduire la variance des données, en réduisant le nombre de points aberrants correspondant aux sujets non motivés ou non concentrés (Camerer et Hogarth [1999]) et en éliminant les erreurs d'inattention (Tversky et Kahneman [1992]).

Notons cependant que, indépendamment même des difficultés liées à la définition d'une norme de performance et évoquées dans l'introduction, l'interprétation des données n'est pas toujours sans ambiguïté. Ainsi, Goeree et Holt [2001] montrent qu'un changement dans la structure des paiements peut modifier les comportements au point qu'ils deviennent totalement contradictoires avec la prédiction théorique testée (l'équilibre de Nash en l'occurrence) alors qu'ils étaient initialement en accord avec elle. Cependant, dans la mesure où il n'y a pas de distinction entre structure des paiements dans les jeux eux-mêmes et 
structure des incitations (puisque les sujets reçoivent systématiquement les sommes impliquées dans les jeux), il est impossible de dire si ce sont les incitations finales et/ou les montants en jeu dans l'expérience elle-même (indépendamment, éventuellement, de ce que les sujets perçoivent effectivement par le biais de l'incitation monétaire) qui ont eu un impact sur les résultats.

\subsubsection{Les incitations inutiles}

Un grand nombre de résultats expérimentaux attestent de l'impact marginal des incitations sur le comportement des sujets. Ces résultats battent en brèche l'idée selon laquelle les observations empiriques contraires aux prédictions théoriques standards (et notamment les fameux "paradoxes" expérimentaux) seraient liées aux faiblesses de la méthode expérimentale en général et à l'absence d'incitations adéquates en particulier.

Ainsi, les psychologues Kogan et Wallach [1964] et Slovic [1969] montrent que la mise en place d'incitations a peu d'impact sur le processus cognitif à l'œuvre chez les sujets. Lichtenstein et Slovic [1973] soulignent également que les incohérences manifestées par de vrais joueurs jouant leur propre argent dans le cadre de mises importantes sont similaires à celles d'étudiants ne procédant qu'à des choix hypothétiques. Il en est de même pour Tversky et Kahneman [1992], qui observent que les violations du modèle d'espérance d'utilité (aversion aux pertes, effet de conséquence commune ou de proportionnalité) sont mises en évidence aussi bien en présence qu'en l'absence d'incitations monétaires

Le premier résultat du même acabit obtenu par des économistes est celui mis en évidence (bien malgré eux !) par Grether et Plott [1979], qui montrent que les incitations ne suppriment pas le phénomène de renversement des préférences (ce dernier s'avérant même plus massif lorsque les choix sont réels). De même, pour ce qui est du caractère bayésien ou non de la 
révision des croyances, les sujets incités financièrement n'apparaissent pas plus 'rationnels' que les autres (Grether [1980]). Enfin, citons l'étude exemplaire de Conlisk [1989]. Lorsque ce dernier reproduit l'expérience d'Allais [1953] en la modifiant sur deux points (recours à des conséquences très faibles $-25,5$ et 0 dollars - et à des incitations réelles), il ne réplique pas le fameux paradoxe. Pour tenter de départager les deux causes possibles de ce résultat, Conlisk mène une autre expérience dans laquelle il conserve les mêmes petits montants mais supprime l'incitation. Le résultat précédent est confirmé, ce qui suggère que c'est davantage la saillance psychologique et cognitive et le réalisme des montants en jeu que leur caractère réel ou hypothétique qui jouent. Les incitations seraient donc secondaires.

Camerer [1989] montre également que la fiabilité des réponses, l'attention accordée à la tâche ou encore le pourcentage de sujets qui choisissent l'alternative la moins risquée dans des choix entre loteries ne sont pas sensibles à la présence ou à l'absence d'incitations (voir aussi Bonner, Young et Hastie [1996] et Di Mauro et Maffioletti [2000]). Dans leur méta-analyse, Bonner et al. [2000] trouvent que les incitations monétaires ne conduisent à une amélioration significative de la performance que dans $50 \%$ des études (nous renvoyons à cet article pour les nombreuses références témoignant de résultats pour le moins contradictoires). Mellers, Weiss et Birnbaum [1992] obtiennent 36\% de violations de la dominance stochastique en présence d'incitations réelles et $45 \%$ lorsque les choix sont hypothétiques, mais la différence n'est pas significative (voir aussi Mellers, Berretty et Birnbaum [1995]). Tversky et Kahneman [1986] mènent un grand nombre d'expériences en introduisant ou non des incitations réelles et montrent que ces dernières n'ont jamais d'impact. Rubinstein [2001] s'élève contre le caractère prétendument nécessaire des incitations monétaires en faisant état d'un grand nombre d'expériences de théorie des jeux dans lesquelles elles n'ont pas d'incidence. Hilton [2001] va plus loin : dans la mesure où même les opérateurs financiers de Wall Street, habitués à manipuler chaque jour des millions de dollars, manifestent des biais 
cognitifs importants, il ne voit pas comment les incitations (et l'apprentissage) offertes dans le cadre d'une expérience pourraient éliminer ces anomalies.

En fait, les incitations semblent affecter la variance des données (le bruit contenu dans les réponses) plutôt que leur teneur. L'étude de Battalio, Kagel et Jiranyakul [1990] souligne que si l'on observe bien des différences quantitatives selon la procédure expérimentale utilisée, les conclusions sont en revanche qualitativement similaires. Les études rapportées par Smith et Walker [1993] suggèrent également, soit que les incitations ne changent pas grand chose, soit qu'une rémunération élevée peut réduire, sans les éliminer, le bruit et/ou les déviations par rapport aux prédictions théoriques conventionnelles. Kagel et Roth [1995] vont plus loin dans le constat et concluent que, quel que soit le contexte de décision (jeu de l'ultimatum, enchère, décision individuelle), ni la taille des incitations ni le fait de lier l'incitation à la performance n'ont d'impact substantiel et systématique sur le comportement observé.

En outre, là encore, l'ambiguïté existe quant à l'interprétation de certains résultats. Ainsi, l'étude de Kroll, Levy et Rapoport [1988] montre que des incitations plus élevées, parce qu'elles augmentent l'aversion au risque des sujets, les conduisent à choisir des portefeuilles contenant une proportion plus importante d'actif non risqué et donc plus proches du portefeuille optimal. Un lien positif se dessine donc en apparence entre incitation et performance (en termes d'optimalité de la décision prise) mais le lien de causalité n'est pas direct, il transite par l'aversion au risque ${ }^{14}$. Cette étude est pourtant abondamment citée comme preuve de ce que des incitations plus élevées améliorent la performance.

Pour finir, notons que la rémunération forfaitaire peut s'avérer plus efficace que les incitations monétaires au sens strict. Church [1993] rapporte à cet égard un résultat (comparatif) intéressant concernant le taux de réponse à un questionnaire : le fait d'offrir aux sujets une

\footnotetext{
${ }^{14}$ Or rien ne prouve que le niveau d'aversion au risque, plus élevé dans le cas fortement incité, soit pour autant plus proche des préférences réelles des sujets.
} 
rémunération lors de l'envoi du questionnaire - et donc de façon inconditionnelle - augmente leur taux de réponse de 20\% (par rapport à une situation sans rémunération du tout) alors que la mise en place d'une rémunération contingente au remplissage du questionnaire laisse le taux de réponse inchangé.

\subsubsection{Les incitations nuisibles}

$\mathrm{Au}$ terme de cette première étape, l'impact des incitations monétaires sur la performance apparaît mitigé. On peut aller plus loin et montrer qu'il peut même être négatif ${ }^{15}$. Ainsi, Titmuss [1970] montre que le fait de payer les donneurs de sang affecte négativement leur disposition à le faire ${ }^{16}$. Dans le même esprit, il apparaît que les élèves qui collectent des dons pour des œuvres de charité sont moins efficaces quand on les rétribue modérément que quand on ne les dédommage pas du tout ${ }^{17}$ (Gneezy et Rustichini [2000]). Wilson et al. [1981] montrent que le fait de payer ou de ne pas payer des étudiants pour résoudre une énigme intéressante affecte le temps libre que ces derniers vont passer dessus (les étudiants rémunérés y consacrent moins de temps). Kohn [1993] s'intéresse aux programmes visant à faire perdre du poids, arrêter de fumer ou attacher sa ceinture de sécurité, dont certains offrent une récompense et d'autres non. A court terme, ce sont les programmes incitatifs qui suscitent le comportement le plus conforme, mais ce n'est plus le cas à long terme.

S'intéressant aux conditions de mise en place d'une décharge de déchets nucléaires infrastructure publique qui génère a priori des externalités négatives, Frey et Oberholzer-Gee

\footnotetext{
${ }^{15}$ Notons cependant la position dissonante de Kunz et Pfaff [2002], qui considèrent d'une part qu'un biais de publication existe en faveur des résultats contraires aux incitations monétaires et d'autre part que la présentation des résultats n'est pas toujours objective (voir leur relecture de Deci, Koestner et Ryan [1999a]).

${ }^{16}$ Solow et Arrow considèrent quant à eux que les effets de l'incitation monétaire s'ajoutent à ceux du don altruiste. Au total, le marché du sang serait un marché comme les autres : lorsque le prix augmente, la quantité totale offerte augmente également (demande normale).

${ }^{17}$ En revanche, une rétribution importante réveille en eux l'homo oeconomicus. Cependant, dans la mesure où il est évidemment difficile de développer massivement un niveau élevé de rétribution, la leçon de ce résultat est qu'il vaut mieux ne pas introduire de rémunération du tout.
} 
[1997] constatent que la majorité des habitants acceptent l'installation d'une telle décharge dans leur secteur, et ce malgré une opinion plutôt négative quant aux risques épidémiologiques qu'elle fait planer. Mais quand on demande aux mêmes individus s'ils sont disposés à accepter l'installation d'une telle décharge sachant que les pouvoirs publics ont décidé de les dédommager, alors le taux d'acceptation tombe de 50.8 à $24.6 \%$, et le niveau de dédommagement n'a pas d'influence.

Face aux résultats empiriques pour le moins ambigus dont nous venons de donner un aperçu, deux stratégies se sont déployées au cours des années 1990. La première, conservatrice, vise à réaffirmer le caractère indispensable des incitations monétaires. Elle attribue donc les "mauvais" résultats empiriques à une mise en œuvre inadéquate des incitations en pratique : leur montant serait trop faible et la procédure incitative mal choisie (section 3). La seconde stratégie, plus ouverte mais aussi plus profonde, souligne la fragilité de la relation entre incitation, effort et performance : les incitations ne stimulent pas toujours l'effort et, même lorsque c'est le cas, l'incidence d'un effort accru sur la performance n'est pas toujours positive (section 4).

\section{La faible incidence des incitations en pratique : l'inadéquation de la procédure incitative}

Dans le cadre de la stratégie conservatrice, deux points surtout ont été soulevés, qui attribuent la relative inefficacité des incitations en pratique à la manière dont elles sont mises en œuvre

- ce qui permet de préserver le postulat selon lequel elles sont nécessaires à une expérimentation de qualité. Sont ainsi critiquées l'insuffisance des incitations mises en œuvre (3.1.) et l'inadéquation des procédures utilisées (3.2.). 


\subsection{Le montant insuffisant des incitations}

Le niveau des incitations mises en œuvre en pratique serait trop faible pour qu'elles jouent un rôle significatif (Jenkins et al. [1998]). Selon Smith [1982] et Smith et Walker [1993], elles doivent en effet être suffisamment élevée pour compenser les coûts subjectifs supportés par le sujet (analyse coût-bénéfice) ou pour amorcer une relation de coopération avec l'expérimentateur .

En fait, l'étude de Kachelmeier et Shehata [1992], qui se donne les moyens de tester l'impact d'incitations très élevées en termes réels (puisqu'elle est menée en Chine, où le coût de la vie est bien plus faible) débouche sur une conclusion mitigée ${ }^{18}$ : les incitations ne sont ni suffisantes ni nécessaires pour assurer la coopération et le sérieux des sujets et en tout état de cause, elles ne sont efficaces que si elles sont très importantes, ce qui exclut d'emblée leur mise en œuvre dans des pays à niveau de vie élevé. D'autres études du même acabit, menées en Indonésie (Cameron [1999]), en République slovaque (Slonim et Roth [1998]) ou encore en Russie (Fehr et Tougareva [1995]) débouchent sur des résultats également peu concluants.

L'étude menée par Holt et Laury [2003] semble en revanche établir que, s'il n'y a pas de différence (en termes d'aversion au risque en l'occurrence) entre choix hypothétiques et choix réels lorsque les montants en jeu sont faibles, cette différence devient significative lorsque les enjeux deviennent plus importants ${ }^{19}$. Les incitations élevées, et elles seulement, auraient donc un impact. Notons cependant que les montants 'importants' utilisés par les auteurs sont de l'ordre de 200 dollars et qu'ils sont donc en réalité très modérés (en revanche, les montants faibles sont vraiment très faibles, ce qui rend leur signification économique discutable). En

\footnotetext{
18 Notons cependant qu'une étude similaire menée auprès de paysans indiens (Binswanger [1980], [1981]) concluait à un impact positif du niveau d'incitation monétaire sur l'attitude vis-à-vis du risque.

${ }^{19}$ Cependant, ce résultat pourrait être dû à l'ordre (en l'occurrence celui des montants croissants) dans lequel les séries de montants ont été proposées aux sujets. Harrison et al. [2003] montrent que des effets d'ordre sont effectivement à l'oeuvre, qui réduisent de moitié les effets de taille des montants établis par Holt et Laury [2003].
} 
outre, dans la mesure où les sujets savent qu'ils vont être confrontés à des choix hypothétiques et à des choix réels - et s'ils se comportent en homo oeconomicus - ils peuvent choisir d'allouer la plus grande partie de leur effort cognitif aux questions réelles, à plus fort enjeu, et de répondre de manière plus désinvolte aux questions hypothétiques.

Signalons pour conclure que sur un plan théorique, des incitations élevées ne sont pas nécessairement plus efficaces. En effet, la perspective d'une richesse accrue peut dissuader l'effort au lieu de le stimuler et, si l'individu souffre d'un manque d'estime de soi, le sentiment de ne pas arriver à atteindre le niveau de performance requis peut définitivement le décourager (voir Bonner et Sprinkle [2002] pour des références). L'existence d'effets potentiellement contradictoires empêche de trancher la question sur le plan théorique en même temps qu'elle contribue à expliquer la diversité des résultats empiriques obtenus.

\subsection{Une procédure incitative inadéquate}

Un deuxième argument utilisé pour récuser les résultats défavorables aux incitations touche à la validité de la procédure incitative utilisée. La procédure la plus adaptée dépend de la nature (individuelle ou interactive) de la décision qui fait l'objet de l'expérience (voir Ohana [2004] pour des considérations concernant ce qu'il appelle la méthode stratégique et qui concerne les expériences interactives). Nous prendrons ici l'exemple de la procédure le plus souvent utilisée en matière de décision individuelle dans le risque et qui a fait l'objet d'une littérature abondante. Il s'agit du 'random-lottery incentive system', qu'on pourrait traduire par 'système de loterie aléatoire'.

Cette procédure consiste à faire jouer pour de vrai une des situations de l'expérience : au début de celle-ci, on informe le sujet que, à l'issue de l'expérience, une des situations de choix rencontrées auparavant sera sélectionnée au hasard (d'où le nom de 'random lottery') et que le 
choix qu'il aura fait à cette occasion décidera de sa rétribution pour l'expérience tout entière. Cette procédure présente une vertu incitative dans la mesure où, ne sachant pas a priori quelle situation sera tirée au sort, le sujet a intérêt à toujours répondre la vérité et à révéler ses vraies préférences, faute de quoi il risque de ne pas recevoir la meilleure rétribution possible. La procédure de la loterie aléatoire constitue donc bien un mécanisme révélateur au sens de la théorie des contrats. Ce système incitatif encourage en outre les sujets à traiter chaque tâche comme une tâche unique, ce qui permet d'éviter les effets de point de référence et de richesse accumulée qui interviendraient si les sujets étaient payés en fonction de leur performance sur chaque question (le sujet pourrait alors intégrer la somme gagnée précédemment avant de faire le choix suivant).

Pourtant, la capacité de cette procédure à générer des données fiables a été largement discutée. Deux types de critiques, l'une théorique et l'autre pratique, lui ont été adressées.

\subsubsection{La critique théorique}

Une première objection, de nature théorique, a été soulevée par Holt [1986]. Ce dernier considère en effet que les incitations monétaires sont si puissantes qu'elles vont amener l'individu à traiter l'ensemble des situations de choix comme un tout au lieu d'aborder chaque question indépendamment. C'est ce qu'il appelle effet de contamination. Or, s'il est équivalent de considérer les tâches comme indépendantes ou comme faisant partie d'un tout lorsque le décideur est maximisateur d'utilité, cette équivalence tombe lorsque l'indépendance est relâchée, l'effet de contamination engendrant alors un biais qui rend la procédure aléatoire inefficace.

Plus précisément, le théorème établi par Holt dit que si les sujets traitent l'ensemble de l'expérience comme un problème de choix unique, que l'axiome d'indépendance n'est pas 
vérifié et que les loteries composées sont réduites en des loteries simples par le calcul des probabilités (c'est-à-dire que l'axiome de réduction des loteries composées est vérifié), alors la procédure aléatoire peut échouer à révéler les vraies préférences. Autrement dit, si l'indépendance est violée mais que la réduction des loteries composées l'est également, alors il est impossible de conclure. Or Starmer et Sugden [1991] montrent que les sujets violent à la fois l'axiome d'indépendance (à travers l'effet de conséquence commune) et l'axiome de réduction des loteries composées. Cela n'exclut pas, naturellement, que la procédure aléatoire soit soumise à d'autres biais ; cependant, les réponses obtenues par les auteurs à l'aide de la procédure aléatoire ne semblent pas très différentes de celles obtenues à l'aide d'autres procédures.

Davis et Holt [1993] émettent des réserves concernant l'étude de Starmer et Sugden, mais les conclusions de cette dernière sont réaffirmées et affinées par Beattie et Loomes [1997]. Ces derniers vont en effet plus loin en menant une nouvelle expérience mettant en jeu six groupes de sujets dans lesquels la nature et la taille des incitations varient (forfait de participation, procédure aléatoire en présence de plusieurs choix, paiement correspondant à la performance sur une seule et unique tâche). A l'issue de cette expérience, les auteurs montrent que dans les problèmes simples de choix entre loteries, la saillance des incitations n'a pas d'impact sur l'aversion au risque. En revanche, quand on sort de ce cadre (en introduisant une dimension temporelle notamment), des effets systématiques et significatifs apparaissent.

\subsubsection{La critique pratique}

Un second argument avancé à l'encontre de la procédure incitative aléatoire met cette fois (et à rebours de l'argument précédent) l'accent sur la dilution des incitations qui résulte de la procédure. Selon l'argument de Smith [1982], la rétribution doit être suffisamment importante 
pour que le sujet n'économise pas son effort mental. Dans le cas présent, c'est donc la rétribution aléatoire (le gain espéré) qui doit être suffisamment élevée. Or, le coût d'un comportement irréfléchi ou d'une erreur (par exemple si l'individu choisit 19 fois sur 20 l'alternative qui maximise son gain espéré mais retient la mauvaise alternative lors du 20 ème choix) est a priori très faible, car la probabilité que le problème pris au hasard à l'issue de l'expérience soit celui pour lequel le sujet a "mal" répondu est elle-même très faible ${ }^{20}$. Le gain supplémentaire attendu d'un effort cognitif accru (ou le coût d'opportunité d'une absence d'effort) peut alors être considéré par le sujet comme insuffisant pour justifier cet effort et l'amener à révéler ses véritables préférences.

Wilcox [1993] émet l'hypothèse que plus les incitations seront diluées dans une procédure de loterie aléatoire, moins les heuristiques utilisées par les sujets seront correctes, et il valide cette hypothèse sur des loteries composées (mais pas sur des jeux simples). De même, Harrison [1994] soutient que pour une tâche donnée, les réponses obtenues avec la procédure de loterie aléatoire contiennent plus d'erreur que celles obtenues avec la procédure de choix réel unique. Cependant, son étude repose sur un échantillon trop petit pour que des tests statistiques puissent véritablement discriminer entre les différences observées. Enfin, selon Davis et Holt [1993], la dilution des incitations dans la procédure aléatoire rend les sujets plus joueurs que s'ils étaient incités sur un choix unique mais ce résultat n'est pas confirmé par Beattie et Loomes [1997].

Afin d'étudier la pertinence empirique des deux critiques théorique et pratique (et le cas échéant de pouvoir discriminer entre elles), Cubitt, Starmer et Sugden [1998] font l'hypothèse que les véritables préférences sont celles manifestées dans le cadre d'une expérience qui comprend une tâche unique et dont le sujet sait qu'il la joue pour de vrai ('real-choice

\footnotetext{
${ }^{20}$ De façon générale, il est judicieux d'empêcher le sujet de déterminer l'espérance (faible en général) de gain. On peut ainsi choisir de dévoiler le montant des gains potentiels mais taire le nombre de candidats ou de choix sur la base desquels se fera le tirage aléatoire, pour éviter que le gain éventuel (élevé) ne soit transposé en gain espéré (faible).
} 
experiment'). Toute procédure incitative conduisant à des choix différents de ceux opérés dans ce cadre est alors considérée comme biaisée. Au terme d'une série de 3 expériences dans lesquelles ils prennent toutes les précautions pour éviter l'expérience à tiroirs, les auteurs ne mettent en évidence ni effet de contamination (argument de Holt) ni effet de dilution (argument de Harrison).

A ce stade de l'analyse, il paraît difficile d'attribuer les résultats contradictoires et/ou mitigés obtenus dans la littérature en ce qui concerne l'impact des incitations sur la performance à des défaillances dans la mise en œuvre de ces incitations. On pourrait certes évoquer le rôle d'autres dimensions de la procédure incitative qui pourraient également affecter l'efficacité de cette dernière, par exemple le moment où la rémunération est remise au sujet, le fait que le contrat d'incitation incorpore ou non une norme explicite de performance, ou encore le fait que ce contrat soit présenté en termes positifs (bonus) ou négatif (pénalité) (Bonner et Sprinkle [2002] ; voir aussi Fehr et Falk [2002]). Il est cependant peu probable que ces dimensions mineures soient responsables de l'ampleur des effets observés. Il convient donc d'envisager d'autres explications.

4. La faible incidence des incitations en pratique : la fragilité de la relation incitation - effort - performance

$\mathrm{Au}$ terme de leur méta-étude méticuleuse, Camerer et Hogarth [1999] concluent que les incitations n'améliorent la performance moyenne que dans les tâches de jugement où l'effort répond aux incitations et la performance à l'effort. Pour un grand nombre de tâches en revanche, les incitations sont inefficaces, soit parce qu'elles n'ont aucun effet sur l'effort fourni (4.1.), soit parce que la performance ne dépend pas de l'effort (4.2.). 


\subsection{L'effort ne dépend pas des incitations}

Si les incitations ont apparemment peu d'incidences sur l'effort fourni par les sujets, c'est probablement parce qu'elles ont en réalité des effets variés, qui jouent simultanément et sont éventuellement contradictoires, de telle sorte que, parfois, leur effet net soit nul (Bonner et Sprinkle [2002] ; Guzzo, Jette et Katzell [1985]). Par exemple, il arrive que les incitations se traduisent par la fixation de buts plus élevés, par un engagement plus soutenu ou une efficacité personnelle accrue, mais ce n'est pas toujours le cas (Lee, Locke et Phan [1997]). Il faut donc rechercher les variables médiatrices de la relation entre incitations et effort. C'est l'objet de l'étude de Bonner et Sprinkle [2002], qui étudie l'influence d'un grand nombre de variables liées soit à la tâche elle-même (complexité, présentation, caractère plus ou moins attractif et enfin mode de réponse), soit à l'environnement expérimental (objectifs fixés et normes de performance, pression temporelle, retour d'expérience par exemple), soit encore à l'individu (aptitudes, besoin d'achèvement, arrière-plan culturel). Nous ne détaillerons pas ici les résultats de ces analyses, trop riches pour que la notion même de synthèse ait un sens. Quoi qu'il en soit, le fait que l'expérimentateur ait une prise sur un grand nombre de ces variables constitue pour lui une invitation à être conscient de ses choix méthodologiques et de leurs implications et à élaborer ses protocoles en connaissance de cause.

Si les psychologues et certains économistes (voir par exemple la position de Kreps [1997]) jugent inutile la mise en place d'une procédure incitative, c'est d'une part parce que cette dernière complique les instructions (Tversky et Kahneman [1992]) et d'autre part et surtout parce que les sujets sont supposés être coopératifs et intrinsèquement motivés à fournir l'effort cognitif requis et à révéler leurs véritables préférences (Camerer [1995]). On appelle motivation intrinsèque la satisfaction que l'individu retire de la tâche et qui le conduit à 
s'engager dans l'activité en l'absence de toute incitation ou menace extérieure (Ryan et al. [1996]. Cette satisfaction peut être liée à la tâche elle-même (objectivement intéressante) et/ou à la psychologie de l'individu (disposé à trouver la tâche intéressante, voir Ryan et Deci [2000]). Cette dernière recouvre en particulier des caractéristiques telles que le besoin d'autonomie, le sens de la réciprocité et la volonté de coopérer, ou encore le besoin de reconnaissance (de ses compétences notamment) ${ }^{21}$. On définit au contraire la motivation extrinsèque comme celle résultant de facteurs externes à la tâche elle-même. Ces facteurs relèvent soit de la carotte (rémunération, délégation, promesses), soit du bâton (surveillance, menace, sanction) (Bénabou et Tirole [2003]). On peut alors effectuer une tâche sans ressentir d'intérêt particulier à son égard voire en dépit d'un désintérêt important, si on y est incité (de manière plus ou moins coercitive) par des instruments externes (Ryan et Deci [2000]). Les incitations monétaires abordées ici appartiennent naturellement à cet ensemble d'outils.

L'idée sous-jacente aux développements théoriques autour du concept de motivation intrinsèque est que les deux types de motivation (intrinsèque et extrinsèque, monétaire notamment) seraient finalement substituables, la motivation intrinsèque s'avérant même plus efficace que les incitants externes pour assurer l'effort et la performance des sujets. Contrairement à l'hypothèse standard selon laquelle l'effort est source de désutilité et doit donc être suscité de manière exogène, que ce soit par la carotte ou le bâton, la fierté d'accomplir telle ou telle tâche, le sentiment d'être utile ou de contribuer à quelque chose peuvent faire de l'effort un argument positif de l'utilité des individus (Kreps [1997]). Plus spécifiquement, selon Loewenstein [1999] ou Henrich [2001], les sources non-monétaires de la motivation, qu'elles soient sociales (statut) ou affectives (émotions), sont plus importantes que - et peuvent l'emporter sur - les incitations financières.

\footnotetext{
${ }^{21}$ Notons que le concept de motivation intrinsèque fait toujours l'objet de discussions et que son contenu est à géométrie variable. Le lecteur intéressé se référera à Ryan et Deci [2000] et Fehr et Falk [2002] pour des détails conceptuels et à Kunz et Pfaff [2002] pour une analyse critique du concept et de sa mesure empirique, pour une
} 
L'argument de la motivation intrinsèque contribue à expliquer que l'incidence des incitations sur l'effort et la performance dans les expériences d'économie soit mitigée. Dans le cadre d'une expérience, on peut en effet supposer que les sujets recrutés (le plus souvent sur la base du volontariat) vont trouver en eux-mêmes les raisons de fournir un effort, que ce soit en raison d'un intérêt intellectuel pour l'objet de l'expérience, d'un souci de réciprocité vis-à-vis de l'expérimentateur ou encore d'un besoin de reconnaissance (par ce dernier et par l'institution en général). Si l'on retient cette hypothèse, les incitations monétaires apparaissent largement inutiles, ce qui veut dire qu'elles sont également inutilement coûteuses.

\subsection{L'effort n'améliore pas toujours la performance}

Même dans l'hypothèse où un sujet incité financièrement en vient à fournir un effort plus important, cet effort accru ne se traduit pas nécessairement par une performance plus élevée, notamment si cette dernière est liée à la mise en œuvre d'un principe de choix ou de jugement rationnel tel que la règle de Bayes. De façon plus générale, l'incitation monétaire peut contribuer à augmenter l'effort cognitif de l'individu, mais certainement pas ses capacités cognitives ou son degré de rationalité intrinsèque (Tversky et Kahneman [1992] ; Camerer et Hogarth [1999]). En particulier, il ne faut pas attendre des incitations qu'elles améliorent l'adhésion aux axiomes de rationalité (Camerer [1995]) : soit les sujets ont des préférences bien formées qu'ils ont de fortes chances d'exprimer de façon fiable, qu'ils soient payés en fonction de leurs réponses (incités) ou non (car on ne voit pas quel intérêt ils auraient à travestir leurs véritables préférences), soit leurs préférences ne sont pas bien formées et il est peu probable que le fait d'être rémunéré change quelque chose. En tout état de cause, il est peu vraisemblable que les sujets soient si sophistiqués et/ou paresseux qu'ils ne se mettent à

présentation des théories cognitive et psychologique sous-jacente et pour de nombreuses références. Nous 
faire des calculs ou à révéler leurs préférences que lorsqu'ils reçoivent une incitation ${ }^{22}$. Comme le souligne Camerer [1992], le fait que les rats exhibent les mêmes comportements que les humains tend à suggérer que l'incapacité des individus à se montrer rationnels est davantage cognitive et perceptuelle que motivationnelle et liée à un déficit d'incitations. Le problème n'étant pas lié à un effort initial insuffisant mais simplement au fait que cet effort est sans effet sur la performance, les incitations monétaires s'avèrent alors inutiles.

Selon Camerer et Hogarth [1999], cette distension du lien entre effort et performance est la raison pour laquelle, dans les tâches les plus étudiées par les économistes (échange sur un marché, négociation et choix entre des loteries), les incitations ne changent pas substantiellement le comportement moyen - même si elles réduisent la variance des réponses en limitant, comme on l'a vu plus haut, le nombre de points aberrants correspondant aux sujets non motivés ou non concentrés. Or les auteurs soulignent que d'autres moyens moins coûteux (statistiques en particulier) permettent de réduire l'influence des points aberrants. En outre, dans la mesure où l'incidence des incitations s'avère comparable à celle d'autres variables telles que l'expérience ou l'habileté et interagit avec elles, il apparaît excessif de se focaliser sur les seules incitations. En particulier, la substituabilité entre expérience et incitations ouvre la possibilité de remplacer les incitations monétaires par le recours à des sujets expérimentés.

Nous avons vu que, face à l'impact pour le moins mitigé des incitations monétaires en pratique, deux stratégies se sont déployées. La première tente de sauver sur un plan théorique les incitations en mettant en cause la manière dont elles sont instaurées en pratique. Au terme de notre analyse, cette approche apparaît peu convaincante. La seconde stratégie, plus nuancée, reconnaît la fragilité théorique de la relation entre incitation, effort et performance, les incitations apparaissant non nécessaires, voire non suffisantes, pour garantir l'effort et/ou 
la performance. Nous allons maintenant franchir une nouvelle étape et montrer que les incitations monétaires peuvent être franchement nuisibles, soit parce qu'elles obèrent l'effort des sujets (5.1.), soit parce qu'un effort accru réduit la performance (5.2.).

\section{Des incitations nuisibles à la performance}

\section{1. Les incitations monétaires nuisent à la motivation intrinsèque et à l'effort}

L'argument que nous évoquons ici considère que les incitations monétaires peuvent réduire la motivation intrinsèque à accomplir une activité (Deci [1975] ; Deci et Ryan, [1985] ; Kreps [1997]) et donc nuire à la performance (Kruglanski [1978]). Cet argument s'inscrit dans la réflexion plus large, évoquée plus haut, concernant les interactions entre incitations extrinsèques (non nécessairement monétaires donc) et motivation intrinsèque, mais il va plus loin. L'idée sous-jacente est que ces deux types de motivation sont chacun source d'effort mais que leur introduction simultanée est contre-productive, les incitations monétaires - et les incitations extrinsèques en général - exerçant un effet d'éviction ${ }^{23}$ à l'encontre de la motivation intrinsèque (Frey [1997] ; Deci, Koestner et Ryan [1999a]) et des autres types de motivation (Baron [2000]).

Notons cependant que la validité de cet argument n'est pas absolue. Les incitations extrinsèques peuvent dans certains cas accroître la motivation intrinsèque (de par leur valeur symbolique et leur impact positif sur le sentiment de compétence ou de réciprocité) et en tout état de cause renforcer ses effets. Le débat empirique, évoqué plus haut, entre l'équipe de Deci et celle de Eisenberger repose précisément sur une conception largement opposée des effets

\footnotetext{
${ }^{22}$ A fortiori, dans les cas où il n'y a pas de norme de performance, l'intérêt des incitations semble encore plus discutable (Baron [2001]).

23 "Crowding out effect". On parle aussi de coût caché.
} 
des incitations monétaires sur la motivation intrinsèque (outre les références déjà citées ${ }^{24}$, voir Eisenberger et Armeli [1997]).

Quoi qu'il en soit, l'effet d'éviction peut être dû à différents facteurs. On en citera quelques uns, sans prétendre à l'exhaustivité (voir Frey et Jegen [2001] pour une recension "économiste" de ces approches). Par exemple, l'approche de l'évaluation cognitive (Deci et Ryan [1985]) prédit que les incitations monétaires sont susceptibles de démotiver l'individu si elles sont perçues comme un moyen de contrôle, car elles affectent alors négativement le besoin d'autonomie et le sentiment de compétence (voir Etzioni [1971], qui montre que le contrôle subi dans les entreprises sous la forme d'incitations financières est vécu par les salariés comme aliénant et déshumanisant). Le modèle principal-agent proposé par James Jr [2005] établit ainsi que la motivation intrinsèque est évincée par la rémunération extrinsèque lorsque cette dernière est perçue par l'agent comme un moyen de contrôle sur son activité. Joue en particulier le fait que l'objet de la motivation intrinsèque et la source de l'incitation monétaire soient confondus. Si par exemple l'agent travaille dans l'intérêt du principal qui est aussi celui qui le paie, alors l'introduction d'incitations va être perçue comme un moyen de manipulation et de contrôle. Il y a évidemment là une frontière ténue entre l'incitation perçue comme contrôle, et donc contre-productive, et celle perçue comme un signal de la difficulté de la tâche et comme la reconnaissance d'une compétence, et qui au contraire accroît la motivation intrinsèque, l'estime de soi et l'effort (Bénabou et Tirole [2003]). Cependant, de façon générale, l'effet d'éviction se produira davantage lorsque les intérêts du principal et ceux de l'agent se confondent que lorsque l'agent est intrinsèquement motivé par des valeurs générales, distinctes du principal (James Jr [2005]).

Une autre approche intéressante est celle liée à la théorie de l'auto-perception (Bem [1967] ; voir aussi Fehr et Falk [2002]]. Dans la mesure où l'individu aurait de toutes façons entrepris

\footnotetext{
${ }^{24}$ Eisenberger et Cameron [1996] récusent l'effet d'éviction et en dénoncent le statut de mythe.
} 
l'activité, tout se passe comme si le fait d'introduire une récompense "surdéterminait" son comportement et le conduisait à réévaluer cognitivement les caractéristiques intrinsèques de l'activité ("si l'on éprouve le besoin de m'inciter à la poursuivre, c'est qu'elle n'est pas si intéressante ou agréable que je le croyais au départ"). Lorsque l'individu entreprend une tâche sans être soutenu par des incitations monétaires, il rationalise l'effort mis en œuvre, ce dernier étant perçu comme le reflet du plaisir trouvé dans l'accomplissement de la tâche - ce plaisir stimulant à son tour l'effort. Si des incitations extrinsèques sont mises en place, alors l'individu attribue son effort aux seules incitations et développe une désutilité de l'effort. (James Jr [2005] ; Kreps [1997]). L'individu vit ainsi une sorte de dissonance cognitive entre ses croyances préalables et celles qui lui sont fournies par les incitations monétaires ${ }^{25}$.

Si les étudiants non rémunérés passent davantage de temps libre sur une activité que ceux qui sont incités (Wilson et al. [1981]), c'est parce que l'introduction d'une incitation monétaire tend à réduire l'intérêt pour la tâche. De même, lorsque l'on demande à des enfants d'accomplir des tâches impliquant des compétences verbales (Kruglanski et al. [1971]), ceux à qui aucune rémunération n'a été promise rapportent un intérêt plus grand pour les tâches en question.

Par ailleurs, selon Condry et Chambers [1978], la récompense détourne l'attention de la tâche (qui est un processus) au profit de la rémunération (qui est le produit de ce processus). L'individu est ainsi détourné des bénéfices de long terme de son activité au profit de la rémunération de court terme : il va prendre la décision qui offre la rétribution immédiate la plus élevée, même si elle est dominée à plus long terme. On est proche ici du biais d'aversion aux pertes myope étudié par Benartzi et Thaler [1995]) et susceptible d'expliquer les comportements observés par Kohn [1993] (dans les programmes visant à inciter les individus à perdre du poids ou à attacher leur ceinture de sécurité, certes les sujets rémunérés adoptent à

\footnotetext{
${ }^{25}$ Notons cependant que ce résultat dépend du niveau initial de motivation intrinsèque : si ce dernier est élevé, alors la rémunération ne sera pas forcément nuisible (Cameron, Banko et Pierce [2001]).
} 
court terme le comportement le plus conforme, mais ce n'est plus le cas à long terme). Le modèle de Bénabou et Tirole [2003] étudie précisément les conditions sous lesquelles les incitations extrinsèques vont s'avérer contre-productives à long terme.

On peut également déceler une logique sociale, en termes de cohérence des rôles et de contrat social, sous les comportements adoptés face aux incitations monétaires (Goffman [1974]). Par exemple, on s'attend à recevoir des incitations monétaires dans une relation de nature économique, tandis qu'on internalise le bien-être d'autrui dans le cadre des relations familiales et amicales. En revanche, au sein d'une organisation ou dans le contexte d'une expérience, aucun schéma préétabli ne prévaut. Cependant, le fait de recruter des sujets sur la base du volontariat par exemple les met d'emblée dans une relation de don-contre-don avec l'expérimentateur, et revenir sur cette logique en instaurant des incitations financières peut être perçu ou ressenti par les sujets comme une modification des règles du jeu, qui va les troubler et affecter de manière négative leur comportement (en y introduisant de l'opportunisme par exemple). Fehr et Falk [2002] soulignent l'existence de motivations sociales non monétaires, comme le désir de réciprocité ou celui d'éviter la désapprobation sociale, qui sont puissamment incitatives en soi et peuvent être détruites par les incitations monétaires. Gneezy et Rustichini [2000] évoquent ainsi la possibilité d'un changement de perception du contrat entre principal et agent : en l'absence d'incitations, ce dernier serait fondé sur des normes sociales de coopération et la réciprocité, normes sociales qui seraient perçues comme inutiles dès lors que des incitations sont introduites. De même, Fehr et Gächter [2002] montrent que le fait d'introduire une rémunération conditionnelle à la performance peut, en stimulant l'égoïsme des agents, réduire la propension à la coopération volontaire - qui traduit une forme d'aversion à l'inéquité - dans le cadre d'une relation vendeur-acheteur ${ }^{26}$

\footnotetext{
${ }^{26}$ Cette explication est cependant insuffisante. Intervient également un effet de présentation (et la présence d'un point de référence par rapport auquel les propositions des agents sont évaluées).
} 
Dans le même ordre d'idée, l'intrusion de la transaction monétaire peut entrer en conflit avec les valeurs morales ou le sens civique de l'individu. Ainsi, l'exemple des donneurs de sang développé par Titmuss [1970] et évoqué plus haut montre que la compensation monétaire tend à réduire le sens du devoir civique chez les individus, autrement dit à détruire partiellement leur motivation intrinsèque et à affecter négativement l'effort fourni. C'est le même phénomène qui est à l'œuvre dans l'exemple de la collecte de dons pour des œuvres de charité $(\text { Gneezy et Rustichini [2000] })^{27}$ ou encore dans celui de la décharge de déchets nucléaires (Frey et Oberholzer-Gee [1997]). Bien sûr, ce dernier résultat pourrait être dû au fait que le fait même de se voir proposer un dédommagement est perçu par les populations comme un signal de dangerosité de la décharge et en réduit donc l'acceptabilité. Mais une fois cet effet neutralisé, il apparaît que la motivation intrinsèque liée au sens civique existe bel et bien et qu'elle est négativement affectée par l'intrusion de la transaction monétaire.

Il est important à ce stade de souligner que les valeurs morales et sociales comme les besoins psychologiques ne sont pas nécessairement les mêmes pour tous les individus. Cela signifie en particulier que la motivation intrinsèque ne repose pas sur les mêmes fondements et n'a pas le même degré d'importance pour tous, et donc que l'interaction entre incitations monétaires et motivation va également être à géométrie variable. Certains résultats empiriques mettant en jeu la motivation intrinsèque et défavorables aux incitations ont ainsi été contestés pour avoir été obtenus sur des enfants, dont le degré de socialisation et les capacités cognitives sont différents de ceux des adultes (Kunz et Pfaff [2002]). Les adultes seraient en effet plus habitués aux récompenses et donc susceptibles d'interpréter les incitations davantage comme un indicateur de performance que comme un moyen de contrôle.

\footnotetext{
${ }^{27}$ On renvoie à Fehr et Falk [2002] pour une interprétation plus poussée de ce résultat en termes d'interaction conflictuelle entre normes sociales et incitations financières.
} 
D'autres critiques d'ordre méthodologique (le choix des indicateurs de motivation intrinsèque, la séparation des différents effets) sont au cœur du débat déjà cité. Pour notre part, il ne s'agit nullement de trancher la question générale "les incitations monétaires détruisent-elles la motivation intrinsèque ?". Il s'agit bien, plutôt, de rappeler et souligner le caractère contingent d'une éventuelle réponse et d'inviter à un certain pragmatisme.

\section{2. Les incitations monétaires nuisent à la performance : trop d'effort tue la performance}

Nous avons vu que les incitations pouvaient nuire à l'effort des sujets en attaquant leur motivation intrinsèque. Nous abordons maintenant un argument plus paradoxal, selon lequel les incitations peuvent affecter négativement la performance parce qu'elles conduisent le sujet à fournir un effort trop important.

Ainsi, l'accomplissement de certaines tâches est incompatible avec un effort cognitif trop important, car ce dernier en devient alors artificiel (Camerer et Hogarth [1999]). C'est le cas des tâches qui requièrent beaucoup de capital (capacités, expérience, savoir-faire technique). En effet, le capital ne peut pas être acquis au cours d'une expérience et il peut être contreproductif d'essayer de le remplacer par un travail (effort) trop intensif, car ce dernier est susceptible de conduire l'individu à, par exemple, sur-apprendre des heuristiques ou sur-réagir aux stimuli.

Par ailleurs, les incitations monétaires peuvent induire des distorsions dans la représentation de la tâche par le sujet. Elles peuvent en particulier amener les sujets à rationaliser des choix qu'ils feraient de manière beaucoup plus instinctive dans la vie. En l'occurrence, la réflexion consciente n'est pas nécessaire pour un grand nombre de tâches telles que la perception, l'apprentissage, la mémoire ou encore le langage (Bargh [1996] ; Slovic et al. [2003]) et le recours à un processus conscient de réflexion plutôt qu'à l'intuition risque dans certains cas de 
réduire la pertinence des jugements effectués ou des décisions prises (Haberstroh, Betsch et Aarts [2000] ; Wilson et Schooler [1991]). Par exemple, les incitations monétaires sont susceptibles de renforcer les biais liés aux croyances initiales, tel le biais d'ancrage (Betsch et Haberstroh [2001]).

Les deux sections précédentes ont tenté de montrer que les incitations monétaires n'étaient pas toujours nécessaires dans le cadre d'une expérience et qu'elles pouvaient même être nuisibles aux résultats. Il s'agit maintenant d'aborder un cas particulier de l'expérimentation qui pose de manière plus aiguë encore la question des incitations. Il s'agit de l'expérimentation dans des contextes de pertes.

\section{Des incitations monétaires délicates voire impossibles à mettre en place : le cas particulier de l'expérimentation dans les pertes}

L'expérimentation dans les pertes soulève une difficulté immédiate, d'ordre déontologique, liée à l'impossibilité de faire 'perdre de leur poche' les sujets (6.1.). On a tenté de contourner cette difficulté à l'aide de procédures incitatives sophistiquées, mais ces dernières soulèvent elles-mêmes d'autres difficultés, pragmatiques et théoriques (6.2.).

\section{1. La difficulté éthique et son contournement}

La difficulté fondamentale rencontrée lors de la mise en place d'un système incitatif dans les pertes est d'ordre éthique, dans la mesure où il est difficile de faire perdre des sujets qui font l'effort de participer à une expérience. Cette difficulté n'est pas seulement celle du chercheur, elle implique aussi l'institution à laquelle il appartient. Comme le soulignent Camerer et Hogarth [1999], "it is generally difficult to impose losses or punishments on subjects for 
bureaucratic reasons - university committees that approve protocols involving human subjects strongly object to it." (p. 36)

Plusieurs travaux ont cependant tenté de contourner cette difficulté. Ainsi, Schoemaker [1990] tire au sort 7 sujets qui jouent 'pour de vrai' les loteries rencontrées. Pour les raisons éthiques évoquées ci-dessus, les pertes nettes ne sont en fait pas appliquées à l'issue de l'expérience, mais cela n'est pas connu des sujets avant la fin de l'expérience. La conclusion de Schoemaker est que les données obtenues sur choix réels et sur choix hypothétiques sont très proches, ce qui permet de se contenter de choix hypothétiques. Ce résultat est une bonne nouvelle, car si la stratégie est astucieuse, elle est 'à un coup' et ne peut être reproduite pour des raisons évidentes de crédibilité !

De leur côté, Thaler et Johnson [1990] développent une expérience dans laquelle ils introduisent une faible probabilité de perdre 'pour de vrai' (faible car les paires de choix dotées de gains préalables importants ont davantage de chances de tomber). La procédure présente donc l'avantage de confronter les sujets à des pertes potentielles réelles même si ceux qui perdront vraiment sont rares. Le recrutement se fait sur la base du volontariat parmi des sujets qui ont déjà participé à d'autres expériences. Cependant, seul un tiers des sujets accepte de participer et il s'agit d'une population particulière, caractérisée par un fort goût pour le risque (biais de sélection de l'échantillon). Une autre expérience est alors menée, dans laquelle tous les sujets sont obligés de jouer 'pour de vrai' mais où la probabilité de perdre est réduite à 0.04, où le montant des pertes est également limité, où il est promis aux sujets qu'ils pourront participer à une tâche rémunérée pour 'se refaire' suite à l'expérience et où, enfin, un bonus est accordé à certains des sujets qui auront joué pour de vrai !! Bref, la portée initiale de l'expérience dans les pertes et les vertus incitatives de la procédure sont considérablement réduites par toutes ces précautions. 


\section{2. Une solution discutable : la dotation initiale}

Une fois admis que les sujets ne peuvent pas perdre de leur poche, on peut tenter de contourner ce problème en leur donnant au préalable une dotation sur laquelle ils vont pouvoir perdre. Une telle procédure soulève cependant plusieurs difficultés.

En premier lieu, comme les contraintes budgétaires de l'expérimentateur sont en général serrées, les sommes nettes reversées aux sujets au terme du jeu (la dotation initiale moins les pertes subséquentes) doivent être relativement réduites. Mais dans ce cas, l'incitation monétaire est elle-même réduite, et les sujets peuvent considérer que cela ne vaut pas la peine de se fatiguer pour une incitation aussi faible (effet de dilution de Harrison [1994]).

En fait, on peut générer des gains finaux faibles de deux manières. La première solution consiste à se contenter de pertes relativement réduites, ce qui permet à la dotation initiale d'être faible mais conduit à une perte de réalisme dans la mesure où les pertes auxquelles sont confrontés les agents dans la vie sont souvent importantes. La deuxième solution consiste à introduire des pertes importantes et réalistes, mais il faut alors que la dotation initiale soit ellemême importante, ce qui est susceptible de poser deux problèmes.

La première difficulté est financière : l'expérimentateur doit être capable de payer une somme importante si jamais la procédure aléatoire se traduit par une perte faible. Une solution alternative serait alors de procéder à une incitation 'fractionnée' (les sommes hypothétiques en jeu sont importantes, mais seule une fraction de ces montants est jouée pour de vrai, ou bien seuls certains sujets jouent pour de vrai). Il devient alors possible de découpler les sommes réelles en jeu des sommes hypothétiques présentes dans l'expérience. Mais là encore, la procédure n'est pas exempte de difficultés, d'une part parce que l'effet incitatif est réduit à proportion et surtout parce que les sujets peuvent être incités à réévaluer tous les montants rencontrés au cours de l'expérience en les divisant par le facteur en question, de telle sorte 
qu'en fait ils ne manipulent pas des montants aussi importants que ceux prévus par l'expérimentateur.

La deuxième difficulté est liée à ce qu'on appelle l'effet de 'house money' (Thaler et Johnson, [1990]), susceptible de se produire dans toute expérience qui implique des décisions dans le risque dès lors que les sujets ne sont pas maximisateurs d'utilité. En effet, dans le modèle d'espérance d'utilité (EU), tous les événements sont codés séparément, de telle sorte que des conséquences préalables n'ont aucun effet sur les choix subséquents. En revanche, si les sujets tendent à évaluer les loteries en termes de gains et de pertes par rapport à un point de référence et non en termes de richesse finale comme dans le modèle EU (ce qui est probablement souvent le cas, cf Tversky et Kahneman [1992] par exemple), alors les choix peuvent être affectés par l'existence de conséquences préalables si ces dernières déplacent le point de référence.

Thaler et Johnson (1990) étudient précisément la façon dont la prise de risques est modifiée par les gains et pertes antérieurs. Il apparaît que les conséquences préalables (dotations initiales) sont combinées aux paiements potentiels offerts par les décisions ultérieures et affectent donc ces dernières (un exemple classique est celui des 'sunk costs') ${ }^{28}$. L'effet de 'house money' se traduit donc par un goût pour le risque supérieur en présence d'un gain préalable (donc d'une dotation initiale). Des résultats similaires sont obtenus par Battalio, Kagel et Jiranyakul $[1990]^{29}$, qui soulignent cependant que l'effet de 'house money' peut recevoir d'autres interprétations (en particulier, le fait de gagner au début du jeu peut mettre dans un état d'esprit positif qui favorise une prise de risques ultérieure). Néanmoins, quelle qu'en soit l'interprétation, l'effet de 'house money' est susceptible de modifier les

\footnotetext{
28 "After a gain, subsequent losses that are smaller than the original gain can be integrated with the prior gain, mitigating the influence of loss aversion and facilitating risk-seeking. The intuition behind this effect is captured by the expression in gambling parlance of 'playing with the house money'.[...] The essence of the idea is that until the winnings are completely depleted, losses are coded as reductions in a gain, as if losing some of 'their money' doesn't hurt as much as losing one's own cash." (Thaler et Johnson [1990], p. 657).
} 
comportements et donc d'affecter la fiabilité des données collectées, ce qui rend délicate la mise en place d'une dotation initiale.

On peut cependant envisager une dernière procédure, qui permet de se passer de dotation initiale et donc d'en éviter les biais. C'est celle qui a été proposée par Cohen, Jaffray et Saïd [1987] et reprise par Abdellaoui [1995]. Cette procédure repose sur le principe d'isolement de Kahneman et Tversky [1979], selon lequel les sujets ont tendance à se représenter les alternatives de choix en ignorant leurs caractéristiques communes éventuelles, telles qu'un bonus commun ou même un résultat conditionnel à un événement donné. Ainsi, on annonce aux sujets qu'à la fin de l'expérience quelques uns seront tirés au sort et confrontés au choix qu'ils auront faits dans deux situations (l'une de gains et l'autre de pertes) et on leur précise qu'une somme de 1000 francs, correspondant à la perte maximale possible, sera versée à chacun des sujets tirés au sort. On pourrait peut-être objecter à cette procédure qu'elle reste faiblement incitative, dans la mesure où les sujets évaluent ex ante la probabilité d'être tirés au sort et peuvent adapter leur comportement (problème de dilution des incitations).

Au total, l'impact pour le moins mitigé des incitations en général et les difficultés et biais qu'occasionne leur introduction dans les pertes débouche sur la conclusion, raisonnablement pragmatique, qu'on peut s'en passer dans ce dernier cas. C'est d'ailleurs le parti-pris adopté dans un certain nombre d'études récentes (Fennema et van Assen [1999] ; Abdellaoui, Vossman et Weber [2005] ; Etchart-Vincent [2004]). On peut d'une part faire confiance à la motivation intrinsèque des sujets et d'autre part considérer que les difficultés cognitives que ces derniers peuvent rencontrer lorsqu'ils prennent des décisions dans les pertes ne sont pas solubles dans les incitations monétaires. Ces difficultés sont probablement bien moins liées à un effort intellectuel insuffisant (qui lui-même serait dû à l'insuffisance des incitations) de la part des sujets qu'à la complexité objective des situations rencontrées. En outre, il serait

\footnotetext{
${ }^{29}$ Citons cependant le résultat dissonant de Binswanger [1981], qui montre que les décisions des sujets à qui on donne une dotation initiale sur laquelle ils jouent par la suite ne diffèrent pas de celles qu'ils prennent quand les
} 
contre-productif de les amener à sur-rationaliser leurs choix, chose qu'ils ne feraient certainement pas dans la vie réelle s'ils étaient confrontés à de telles situations.

\section{Conclusion}

$\mathrm{Au}$ terme de cette étude, toute position catégorique concernant les incitations monétaires apparaît irrecevable parce qu'essentiellement dogmatique. La littérature tant théorique qu'empirique invite à la prudence et au pragmatisme : les incitations monétaires ne sont ni nécessaires, ni inutiles, ni nuisibles en soi. Elles vont l'être par rapport à un projet expérimental particulier, car chaque expérimentation présente des caractéristiques propres (en termes de nature de la tâche à accomplir, d'environnement expérimental, de ressources morales et cognitives mobilisées chez les sujets, de caractéristiques des sujets impliqués).

Il est parfois préconisé de construire une base de données permettant de savoir sous quelles conditions la rémunération modifie sensiblement les comportements (Hertwig et Ortmann [2001] ; Ohana [2004]). Selon nous, la mise en forme d'une telle masse d'information serait d'une part inutilement longue et fastidieuse et d'autre part illusoirement rassurante : la frontière entre les différentes catégories ainsi identifiées ${ }^{30}$ serait nécessairement poreuse, ne serait-ce que parce que l'effet pur des incitations est difficilement mesurable et que les études sont souvent difficilement comparables entre elles ${ }^{31}$. En outre, dans la mesure où il existe des substituts non monétaires à la rémunération incitative, il est possible de satisfaire au même objectif méthodologique sans nécessairement recourir à cette dernière.

Bref, selon nous, la seule vraie règle générale à suivre pour un expérimentaliste est de connaître les enjeux sous-jacents à la question des incitations monétaires - nous espérons par

\footnotetext{
paiements ne sont donnés qu'après le jeu.

${ }^{30}$ Les études étant distinguées selon que l'impact des incitations sur la performance y est apparu positif, négatif, nul, impossible à mesurer et/ou à séparer d'autres effets ou que la notion même de performance n'y a pas de sens.
} 
cet article lui permettre d'y accéder au moindre coût - et plus particulièrement ceux qui concernent directement son objet d'étude, afin d'être en mesure de mener ses travaux en connaissance de cause, sans commettre d'imprudence ni d'excès de zèle, et de pouvoir ensuite les insérer dans la littérature existante tout en étant capable de justifier ses pratiques méthodologiques. Concrètement, cette démarche devrait le conduire à utiliser des incitations dans les contextes expérimentaux où les études antérieures ont mis en évidence un effet substantiel de ces dernières, mais à ne pas (se sentir obligé de) le faire quand cet impact est apparu négligeable ou contre-productif ${ }^{32}$.

\footnotetext{
${ }^{31}$ Comme le suggèrent Camerer et Hogarth [1999], il faudrait commencer par exprimer les incitations dans une même unité monétaire, en monnaie constante en particulier, avant d'effectuer des comparaisons.

${ }^{32}$ Dans cette optique, Camerer et Hogarth [1999] invitent les rapporteurs à la modération : "Referees who would reject a paper purely on the grounds that subjects were not paid must cite a preponderance of previous literature establishing that incentives affect behavior meaningfully, in a task similar to that studied in the paper under consideration." (p. 35)
} 


\section{Références}

ABDELLAOUI M. [1995], "Comportements individuels devant le risque et transformation des probabilités", Revue d'Economie Politique, vol. 105, n¹, p. 157-178.

ABDELLAOUI M., VOSSMAN F. et WEBER M. [2005], "Choice-Based Elicitation and Decomposition of Decision Weights for Gains and Losses Under Uncertainty", Management Science, à paraître.

AKERLOF G. [1984], "Gift Exchange and Efficiency Wage Theory: Four Views", American Economic Review, vol. 76, pp. 79-83.

ALLAIS M. [1953], "Le Comportement de l'Homme Rationnel devant le Risque et Critique des Postulats et Axiomes de l'Ecole Américaine", Econometrica, vol. 21, pp. 503-546.

BARGH J. A. [1996], "Principles of Automaticity", in E. T. Higgins et A. Kruglanski (eds.), Social Psychology: Handbook of Basic Principles, Guilford Press.

BARON J. [2000], Thinking and Deciding, Cambridge University Press, Cambridge, UK (3ème Edition).

BARON J. [2001], "Purposes and Methods" (Open Peer Commentary), Behavioral and Brain Sciences, vol. 24, $\mathrm{n}^{\circ}$ 3, p. 403.

BATTALIO R., KAGEL J. et JIRANYAKUL K. [1990], "Testing between alternative models of choice under uncertainty: some initial results", Journal of Risk and Uncertainty, vol. 3 , pp. 25-50.

BEATTIE J. et LOOMES G. [1997], "The Impact of Incentives upon Risky Choice Experiments", Journal of Risk and Uncertainty, vol. 14, pp. 155-168.

BEM D. J. [1967], "Self-Perception: An Alternative Interpretation of Cognitive Dissonance Phenomena", Psychological Review, vol. 74, pp. 183-200.

BENABOU R. et TIROLE J. [2003], "Intrinsic and Extrinsic Motivation", Review of Economic Studies, vol. 70, n 3, pp. 489-520.

BENARTZI S. et THALER R. H. [1995], "Myopic Loss Aversion and the Equity Premium Puzzle", Quaterly Journal of Economics, vol. 110, n 1, pp. 73-92.

BETSCH T. et HABERSTROH S. [2001], "Financial Incentives Do Not Pave the Road to Good Experimentation" (Open Peer Commentary), Behavioral and Brain Sciences, vol. 24, n 3, p. 404.

BINSWANGER H. P. [1980], "Attitudes towards Risk: Experimental Measurement in Rural India", American Journal of Agricultural Economics, vol. 62, pp. 395-407.

BINSWANGER H. P. [1981], "Attitudes towards Risk: Theoretical Implications of an Experiment in Rural India", Economic Journal, vol. 91, pp. 867-890.

BOHM P. [1994], "Time Preference and Preference Reversal among Experienced Subjects: the Effects of Real Payments", Economic Journal, 104, pp. 1370-1378.

BONNER S. E., HASTIE R., SPRINKLE G. B. et YOUNG S. M. [2000], "A review of the Effects of Financial Incentives on Performance in Laboratory Tasks: Implications for Management Accounting", Journal of Management Accounting Research, vol. 13, pp. 19-64.

BONNER S. E. et SPRINKLE G. B. [2002], "The Effects of Monetary Incentives on Effort and Task Performance: Theories, Evidence, and a Framework for Research", Accounting, Organizations and Society, vol. 27, pp. 303-345.

BONNER S. E., YOUNG S. M. et HASTIE R. [1996], "Financial Incentives and Performance in Laboratory Tasks: The Effects of Task Type and Incentive Scheme Type", Department of Accounting, University of Southern California, Los Angeles, CA. 
BROOKSHIRE D. S. et COURSEY D. L. [1987], "Measuring the Value of a Public Good: An Empirical Comparison of Elicitation Procedures", American Economic Review, vol. 77, pp. 554-566.

BURKE M. S. [1996], "An Experimental note on the Allais paradox and Monetary Incentives", Empirical Economics, vol. 6, pp. 17-32.

CAMERER C. F. [1989], "An Experimental Test of Several Generalized Utility Theories", Journal of Risk and Uncertainty, vol. 2, pp. 61-104.

CAMERER C. F. [1992], "Recent Tests of Generalizations of Expected Utility Theory", in W. Edwards (ed.), Utility Theories: Measurements and Applications, Kluwer Academic Publishers, Boston.

CAMERER C. F. [1995], "Individual Decision Making", in J. H. Kagel and A. E. Roth (eds.), Handbook of Experimental Economics, Princeton University Press, Princeton, NJ.

CAMERER C. F. [1997], "Rules for Experimenting in Psychology and Economics and Why They Differ", in Albers et al. (eds.), Understanding Strategic Interactions. Essays in Honor of Reinhard Selten, Springer, pp. 313-327.

CAMERER C. F. et HOGARTH R. M. [1999], "The Effects of Financial Incentives in Experiments: A Review and Capital-Labor-Production Framework", Journal of Risk and Uncertainty, vol. 19, $\mathrm{n}^{\circ}$ 1, pp. 7-42.

CAMERON L. [1999], "Raising the Stakes in the Ultimatum Game: Experimental Evidence from Indonesia", Economic Inquiry, vol. 37, n 1, pp. 47-59.

CAMERON L., BANKO K. M. et PIERCE W. D. [2001], "Pervasive Negative Effects of Rewards on Intrinsic Motivation: The Myth Continues", The Behavior Analyst, vol. 24, pp. 1-44.

CHURCH A. H. [1993], "Estimating the Effects of Incentives on Mail Survey Response Rates: A Meta-Analysis, Public Opinion Quaterly, vol. 57, pp. 62-79.

COHEN M., JAFFRAY J.-Y. et SAÏD T. [1987], "Experimental Comparison of Individual Behavior Under Risk and Under Uncertainty", Organizational Behavior and Human Decision Processes, vol. 39, pp. 1-22.

CONLISK J. [1989], "Three Variants on the Allais Example", The American Economic Review, vol. 79, n 3, pp. 392-407.

CONDRY J. et CHAMBERS J. [1978], "Intrinsic Motivation and the Process of Learning", in M. Lepper et D. Greene (eds.), The Hidden Costs of Reward: New Perspectives on the Psychology of Human Motivation (New York: John Wiley).

COURSEY D. L., HOVIS J. L. et SCHULZE W. D. [1987], "The Disparity between Willingness to Accept and Willingness to Pay Measures of Value", Quaterly Journal of Economics, vol. 102, pp. 679-680.

CUBITT R. P., STARMER C. et SUGDEN R. [1998], "On the Validity of the Random Lottery Incentive System", Experimental Economics, vol. 1, pp. 115-131.

DAVIS D. D. et HOLT C. A. [1993], Experimental Economics, Princeton, NJ: Princeton University Press.

DECI E. L. [1975], Intrinsic Motivation, New York: Plenum Press.

DECI E.L., KOESTNER R. et RYAN R. M. [1999a], "A Meta-Analytic Review of Experiments Examining the Effects of Extrinsic Rewards on Intrinsic Motivation", Psychological Bulletin, vol. 125, pp. 627-668.

DECI E.L., KOESTNER R. et RYAN R. M. [1999b], "The Undermining Effect Is a Reality After All - Extrinsic Rewards, Task Interest, and Self-Determination: Reply to Eisenberger, Pierce, and Cameron (1999) and Lepper, Henderlong, and Gingras (1999)", Psychological Bulletin, vol. 125, $\mathrm{n}^{\circ}$ 6, pp. 692-700.

DECI E. L. et RYAN R. M [1985], Intrinsic Motivation and Self-Determination in Human Behavior, New York: Plenum Press. 
DI MAURO C. et MAFFIOLETTI A.[2000], "Reaction to Uncertainty and Market Mechanisms: Experimental Evidence", Dept of Economics, University of Torino.

EISENBERGER R. et ARMELI S. [1997], "Can Salient Reward Increase Creative Performance Without Reducing Intrinsic Creative Interest?", Journal of Personality and Social Psychology, vol. 72, $\mathrm{n}^{\circ}$ 3, pp. 652-663.

EISENBERGER R. et CAMERON J. [1996], "Detrimental Effects of Reward: Reality or Myth?", American Psychologist, vol. 51, pp. 1153-1166.

EISENBERGER R. et CAMERON J. [1998], "Reward, Intrinsic Interest, and Creativity: New Findings", American Psychologist, vol. 53, n 6, pp. 676-679.

EISENBERGER R., PIERCE W. D. et CAMERON J. [1999], "Effects of Reward on Intrinsic Motivation - Negative, Neutral, and Positive: Comment on Deci, Koestner, and Ryan (1999)", Psychological Bulletin, vol. 125, n 6, pp. 677-691.

EISENBERGER R., RHOADES L. et CAMERON J. [1999], "Does Pay for Performance Increase or Decrease Perceived Self-Determination and Intrinsic Motivation?", Journal of Personality and Social Psychology, vol. 77, n 5, pp. 1026-1040.

ETCHART-VINCENT N. [2004], "Is Probability Weighting Sensitive to the Magnitude of Consequences? An Experimental Investigation on Losses", Journal of Risk and Uncertainty, vol. 28, $\mathrm{n}^{\circ} 3$, pp. 217-235.

ETCHART-VINCENT N. [2005], "Plaidoyer pour un recours éclairé à la méthode expérimentale en économie", en révision.

ETZIONI A. [1971], Modern Organizations, Englewood Cliffs, N.J.: Prentice Hall.

FEHR E. et FALK A. [2002], "Psychological Foundations of Incentives", European Economic Review, European Economic Review, vol. 46, pp. 687-724.

FEHR E. ET GÄCHTER S. [2002], "Do Incentive Contracts Undermine Voluntary Cooperation?", Working paper $n^{\circ} 34$, University of Zurich.

FEHR E. et TOUGAREVA E. [1995], "Do Competitive Markets with High Stakes Remove Reciprocal Fairness? - Evidence from Russia", Discussion Paper, University of Zurich.

FENNEMA H. et VAN ASSEN M. [1999], "Measuring the utility of losses by means of the tradeoff method", Journal of Risk and Uncertainty, vol. 17, $\mathrm{n}^{\circ} 3$, pp. 277-295.

FREY B. S. [1997], Not Just for the Money: An Economic Theory of Personal Motivation, Edward Elgar Publishing, Brookfield, US.

FREY B. S. et JEGEN R. [2001], "Motivation Crowding Theory", Journal of Economic Surveys, vol. $15, \mathrm{n}^{\circ}$ 5, pp. 589-611.

FREY B. S. et OBERHOLZER-GEE F. [1997], "The Cost of Price Incentives: An Empirical Analysis of Motivation Crowding-Out", American Economic Review, vol. 87, pp. 746755.

GIBBONS R. [1997]\}"Incentives and Careers in Organizations", in D. Kreps and K. Wallis, eds., Advances in Economic Theory and Econometrics, vol. II, Cambridge, England: Cambridge University Press.

GINTIS H. [2001], "The Contribution of Game Theory to Experimental Design in the Behavioral Sciences" (Open Peer Commentary), Behavioral and Brain Sciences, vol. 24, $\mathrm{n}^{\circ}$ 3, pp. 411-412.

GNEEZY U. et RUSTICHINI A. [2000], "Pay Enough or Don't Pay at All", Quaterly Journal of Economics, vol. 115, pp. 791-810.

GOERE J. K. et HOLT C. A. [2001], Ten Little Treasures of Game Theory and Ten Intuitive Contradictions", American Economic Review, vol. 914, n 5, pp. 1402-1422.

GOFFMAN E. [1974], Frame Analysis: Essays on the Organization of Experience, Harper, 1974.

GRETHER D. M. [1980], "'Bayes's Rule as a Descriptive Model: The Representativeness Heuristic", Quaterly Journal of Economics, vol. 95, pp. 537-57. 
GRETHER D. M. et PLOTT C. R. [1979], "Economic Theory of Choice and the Preference Reversal Phenomenon", American Economic Review, vol. 69, n4, pp. 623-638.

GUZZO R. A., JETTE R. D. et KATZELL R. A. [1985], "The Effects of Psychologically Based Intervention Programs on Worker Productivity: A Meta-Analysis", Personnel Psychology, vol. 38, pp. 275-291.

HABERSTROH S., BETSCH T. et AARTS H. [2000], "When Guessing is better than Thinking: Multiple Bases for Frequency Judgments", Working Paper, University of Mannheim.

HARLESS D. W. et CAMERER C. F. [1994], "The Predictive Utility of Generalized Expected Utility Theories", Econometrica, vol. 62, nº6, pp. 1251-1289.

HARRISON G. W. [1992], "Theory and Misbehavior of First-Price Auctions", American Economic Review, vol. 79, pp. 749-762.

HARRISON G. W. [1994], "Expected Utility Theory and the Experimentalists", Empirical Economics, vol. 19, pp. 223-253.

HARRISON G. W., JOHNSON E., McINNES M. M. et RUTSTRÖM E. E. [2003], "Risk Aversion and Incentive Effects: Comment", Working Paper, Department of Economics, Moore School of Business, University of South Carolina, USA.

HARRISON G. W., LIST J. A. [2004], "Field Experiment", Journal of Economic Literature, vol. 42, $\mathrm{n}^{\circ}$ 4, pp. 1013-1059.

HARRISON G. W. et RUTSTRÖM E. E. [2004], "Experimental Evidence on the Existence of Hypothetical Bias in Value Elicitation Experiments", in C. R. Plott et V. L. Smith (eds.), Handbook of Experimental Economics Results, New York: Elsevier Press.

HENRICH J. [2001], "Challenges to Everyone: real People, Deception, One-Shot Games, Social Learning and Computers" (Open Peer Commentary), Behavioral and Brain Sciences, vol. 24, $\mathrm{n}^{\circ} 3$, pp. 414-415.

HERTWIG R. et ORTMANN A. [2001], "Experimental Practices in Economics: A Methodological Challenge for Psychologists?", Behavioral and Brain Sciences, 24, pp. 383-451.

HILTON D. J. [2001], "Is the Challenge for Psychologists to Return to Behaviourism?", (Open Peer Commentary), Behavioral and Brain Sciences, vol. 24, n 3, pp. 415-416.

HIRSHLEIFER J. et RILEY J. G. [1992], The Analytics of Uncertainty and Information, Cambridge University Press, Cambridge, UK.

HOLT C. A. [1986], "Preference Reversals and the Independence Axiom", American Economic Review, vol. 76, pp. 508-515.

HOLT C. A. et LAURY S. K. [2003], "Risk Aversion and Incentive Effects", American Economic Review, vol. 92, pp. 1644-1655.

JAMES H. S. [2005], "Why Did You Do That? An Economic Examination of the Effect of Extrinsic Compensation on Intrinsic Motivation and Performance", Journal of Economic Psychology, à paraître.

JENKINS G. D., MITRA A., GUPTA N. et SHAW J. D. [1998], "Are Financial Incentives Related to Performance? A Meta-Analytic Review of Empirical Research", Journal of Applied Psychology, vol. 83, pp. 777-787.

JOBBER D., SAUNDERS J. et MITCHELL V.-W. [2004], "Prepaid Monetary Incentive Effects on Mail Survey Response", Journal of Business Research, vol. 57, pp. 347-350.

KACHELMEIER S. J. et SHEHATA M. [1992], "Examining Risk Preferences Under High Monetary Incentives: Experimental Evidence from the People's Republic of China", American Economic Review, vol. 82 n 5, pp. 1120-1141.

KAGEL J. et ROTH A. (eds.) [1995], The Handbook of Experimental Economics, Princeton, NJ: Princeton University Press. 
KAHNEMAN D. et TVERSKY A. [1979], "Prospect Theory: An Analysis of Decision Under Risk", Econometrica, vol. 47, pp. 263-291.

KOGAN, N. et WALLACH, M. A. [1964], Risk Taking: A study in Cognition and Personality, New York: Holt, Rinehard and Winston.

KOHN A. [1993], Punished by Rewards, New York: Plenum Press.

KREPS D. M. [1997], "Intrinsic Motivation and Extrinsic Incentives", AER Papers and Proceedings, vol. 87, $\mathrm{n}^{\circ}$ 2, pp. 359-364.

KROLL Y., LEVY H. et RAPOPORT A. [1988], "Experimental Tests of the Separation Theorem and the Capital Asset Pricing Model", American Economic Review, vol. 78, pp. 500-519.

KRUGLANSKI A., FRIEDMAN I. et G. ZEEVI [1971], "The Effect of Extrinsic Incentives on Some Qualitative Aspects of Task Performance", Journal of Personality, vol. 39, pp. 608-617.

KRUGLANSKI A. [1978], "Issues in Cognitive Social Psychology", in The Hidden Cost of Reward: New Perspectives on the Psychology of Human Motivation, New Yor: John Wiley.

KÜHBERGER A. [2001], "Why Use Real and Hypothetical Payoffs?" (Open Peer Commentary), Behavioral and Brain Sciences, vol. 24, $\mathrm{n}^{\circ} 3$, pp.419-420.

KUNZ A. H. et PFAFF D. [2002], "Agency Theory, Performance Evaluation, and the Hypothetical Construct of Intrinsic Motivation", Accounting, Organizations and Society, vol. 27, pp. 275-295.

LAZEAR E. [2000], "Performance, Pay and Productivity", American Economic Review, vol. 90, n 5, pp. 1346-1361.

LEPPER, M. R., HENDERLONG, J. et GINGRAS, I. [1999], "Understanding the effects of extrinsic rewards on intrinsic motivation. Uses and abuses of meta-analysis: Comment on Deci, Koestner, and Ryan (1999)", Psychological Bulletin, vol. 125, pp. 669-676.

LEVIN I. P., CHAPMAN D. P. et JOHNSON R. D. [1988], "Confidence in Judgments Based on Incomplete Information: An Investigation using Both Hypothetical and Real Gambles", Journal of Behavioral Decision Making, vol. 1, pp. 29-41.

LICHTENSTEIN S. et SLOVIC P. [1973], "Response-Induced Reversals of Preferences in Gambling: An Extended Replication in Las Vegas", Journal of Experimental Psychology, vol. 101, pp. 16-20.

LOEWENSTEIN G. F. [1999], "Experimental Economics from the Vantage-Point of Behavioural Economics", Economic Journal, 109, pp. 25-34.

MELLERS B. A., WEISS R. et BIRNBAUM M. H. [1992], "Violations of Dominance in Pricing Judgments", Journal of Risk and Uncertainty, vol. 5, pp. 73-90.

MELLERS B. A., BERRETTY P. M. et BIRNBAUM M. H. [1995], "Dominance Violations in Judged Prices of Two- and Three-Outcome Gambles", Journal of Behavioural Decision Making, vol. 8, pp. 211-216.

OHANA M. [2004], "L'expérimentation en économie et en psychologie : une comparaison méthodologique", Revue de Philosophie Economique, ${ }^{\circ} 10$.

ROTH A. [1988], "Laboratory Experimentation in Economics: A Methodological Overview", Economic Journal, pp. 974-1031.

ROTH A. [1993], "On the Early History of Experimental Economics: A Methodological Overview", Journal of the History of Economic Thought, vol. 15, pp. 184-209.

RUBINSTEIN A. [2001], "A Theorist's View of Experiments", European Economic Review, 45, pp. 615-628.

RYAN R. M. et DECI E. L. [2000], "Intrinsic and Extrinsic Motivations: Classic Definitions and New Directions", Contemporary Educational Psychology, vol. 25, pp. 54-67. 
RYAN R. M., SHELDON K. M., KASSER T. et DECI E. L. [1996], "All Goals Are Not Created Equal: An Organismic Perspective on the Nature of Goals and their Regulation", in P. M. Gollwitzer et al. (eds.), The Psychology of Action: Linking Cognition and Motivation to Behavior, pp. 7-26, New York, NY: The Guilford Press.

SCHOEMAKER P. J. H. [1990], "Are Risk Attitudes Related Across Domains and Response modes?", Management Science, vol. 36, n ${ }^{\circ}$, pp. 1451-1463.

SEAL K. H., KRAL A. H., LORVICK J., MCNEES, GEE L. et EDLIN B. R. [2003], "A Randomized Controlled Trial of Monetary Incentives vs. Outreach to Enhance Adherence to the Hepatitis B Vaccine Series Among Injection Drug Users", Drug and Alcohol Dependence, vol. 71, pp. 127-131.

SHAPIRA Z. [2000], "Aspiration Levels and Risk Taking: A Theoretical Model and Empirical Study on the Behavior of Government Bond Traders", Unpublished Manuscript, New York University.

SLONIM R. et ROTH A. [1998], "Learning in High Stakes Ultimatum Games: An Experiment in the Slovak Republic", Econometrica, vol. 66, n 3, pp. 569-596.

SLOVIC P. [1969], "Differential Effects of Real versus Hypothetical Payoffs on Choices Among Gambles", Journal of Experimental Psychology, vol. 80, pp. 434-437.

SLOVIC P., FINUCANE M. L., PETERS E. et McGREGOR D. [2003], "Risk as Analysis and Risk as Feelings: Some Thoughts about Affect, Reason, Risk and Rationality", Risk Analysis, à paraître.

SMITH V. L. [1976], "Experimental Economics Induced Value Theory", American Economic Review, vol. 66, pp. 274-279.

SMITH V. L. [1982], "Microeconomic Systems as an Experimental Science", American Economic Review, vol. 72, pp. 923-955.

SMITH V. L. [1991], "Rational Choice: the Contrast Between Economics and Psychology", Journal of Political Economy, vol. 99, pp. 877-897.

SMITH V. L. et LEVIN I. P. [1996], "Need for Cognition and Choice Framing Effects", Journal of Behavioral Decision Making, vol. 9, pp. 283-290.

SMITH V. L. et WALKER J. [1993], "Monetary Rewards and Decision Cost in Experimental Economics", Economic Inquiry, vol. 31, pp. 245-261.

STARMER C. et SUGDEN R. [1991], "Does the Random-Lottery Incentive System Elicit True Preferences? An Experimental Investigation", American Economic Review, vol. 81, $\mathrm{n}^{\circ} 4$, pp. 971-978.

THALER R. H. et JOHNSON E. J. [1990], "Gambling with the House Money and Trying to Break Even: The Effects of Prior Outcomes on Risky Choice", Management Science, vol. 36, $\mathrm{n}^{\circ}$ 6, pp. 643-660.

TITMUSS R. M. [1970], The Gifts Relationship, London: Allen and Unwin.

TVERSKY A. et KAHNEMAN D. [1986], "Rational Choice and the Framing of Decisions", Journal of Business, vol. 59, n 4 , pp. 251-278.

TVERSKY A. et KAHNEMAN D. [1992], "Advances in Prospect Theory: Cumulative Representation of Uncertainty", Journal of Risk and Uncertainty, vol. 5, pp. 297-323.

van WALLENDAEL L. R. et GUIGNARD Y. [1992], "Diagnosticity, Confidence and the Need for Information", Journal of Behavioral Decision Making, vol. 5, pp. 25-37.

WALLIS W. A. et FRIEDMAN M. [1942], "The empirical derivation of indifference functions", in O. Lange, F. McIntyre and T. O. Yntema (eds), Studies in mathematical economics and econometrics in memory of Henry Schultz, Chicago: University of Chicago Press.

WILCOX N. T. [1993], "Lottery Choice: Incentives, Complexity and Decision Time", Economic Journal, vol. 103, pp. 1397-1417. 
WILSON T. D., HULL J. et JOHNSON J. [1981], "Awareness and Self-Perception: Verbal Reports on Internal States", Journal of personality and Social Psychology, vol. 40, pp. 53-71.

WILSON T. D. et SCHOOLER J. W. [1991], "Thinking Too Much: Introspection Can Reduce the Quality of Preferences and Decisions", Journal of Personality and Social Psychology, vol. 60, pp. 181-192.

LEE T. W., LOCKE E. A. et PHAN S. H. [1997], "Explaining the Assigned Goal-Incentive Interaction: The Role of Self-Efficacy and Personal Goals", Journal of Management, vol. 23, pp. 541-559. 